Boletín de la Sociedad Geológica Mexicana

VOLUMEN 63, NÚM. 2, 2011, P. 183-199

\title{
Estudio petrográfico y geoquímico del Complejo Plutónico El Peñuelo (Cinturón de Intrusivos de Concepción del Oro), noreste de México
}

\author{
Fernando Velasco-Tapia ${ }^{1, *}$, Reneé González-Guzmán ${ }^{1}$, Gabriel Chávez-Cabello ${ }^{1}$, \\ Javier Lozano-Serna ${ }^{1}$, Martín Valencia-Moreno ${ }^{2}$ \\ ${ }^{1}$ Facultad de Ciencias de la Tierra, Universidad Autónoma de Nuevo León, Carretera Linares-Cerro Prieto km 8, 67700 Linares \\ Nuevo León. \\ ${ }^{2}$ Estación Regional del Noroeste, Instituto de Geología, Universidad Nacional Autónoma de México; Luis Donaldo Colosio y Madrid \\ s/n, Campus UNISON, 83000 Hermosillo, Sonora. \\ *velasco@fct.uanl.mx
}

\begin{abstract}
Resumen
El presente estudio reporta información petrográfica y geoquímica del Complejo Plutónico El Peñuelo (CPEP), perteneciente al Cinturón de Intrusivos de Concepción del Oro (CICO, noreste de México). El CPEP es una estructura semi-circular, constituida por tres centros de emplazamiento, ubicada en el extremo oriental del CICO y en la extensión norte de la traza del sistema de fallas Taxco-San Miguel de Allende. Sin embargo, el complejo no fue deformado por la actividad de este sistema de fallas. El CPEP está constituido por rocas intrusivas que varían desde cuarzo monzodiorita a cuarzosienita, siendo ésta última la unidad que cubre la mayor parte de la superficie del complejo. El CPEP se emplazó en rocas sedimentarias marinas del Cretácico superior. La cuarzosienita es cortada por diques de cuarzomonzodiorita y mesosienita porfíritica. Además, esta unidad contiene enclaves microgranulares de monzodiorita distribuidos de forma irregular y diques pegmatíticos cortan al resto de las unidades litológicas. La asociación mineralógica en el CPEP presenta cantidades variables de plagioclasa + feldspato alcalino + cuarzo \pm anfíbol + biotita \pm ortopiroxeno + clinopiroxeno + óxidos de Fe-Ti. Las rocas intrusivas tienen una composición química en $\mathrm{SiO}_{2}=45.7-72.0 \%$, Mg\# = 39.2-60.2 y n-Fe = 0.54-0.73. Presentan patrones de tierras raras, normalizados a condrita, enriquecidos en elementos ligeros $\left[(\mathrm{La} / \mathrm{Yb})_{\mathrm{N}}=6-11\right]$ sin anomalías de Eu. Los diagramas multielementos, normalizados a manto primordial, muestran patrones de enriquecimiento en elementos LILE en relación a los HFSE. Su geoquímica de elementos traza es similar a la de granitoides de alto Ba-Sr: (a) una alta concentración de Ba (= 594-2302 ppm) y $\mathrm{Sr}$ (= 444-2192 ppm); (b) una baja concentración de Y (=10-46 ppm) y Nb (=6-17 ppm); y (c) valores altos para las relaciones $\mathrm{Sr} / \mathrm{Y}(=25-85)$ y La/Yb (= 8.9-16.5). El origen del CPEP se ha relacionado a la fusión parcial de un manto litosférico enriquecido, en condiciones post-orogénicas, seguida de cristalización fraccionada acoplada con asimilación de material cortical.
\end{abstract}

Palabras clave: Noreste de México, granitoides de alto Ba-Sr, magmatismo post-orogénico.

\begin{abstract}
This work presents the petrographic and geochemical data for the El Peñuelo Plutonic Complex (EPPC), which is an intrusive that belongs to Concepción del Oro Intrusive Belt (COIB; Northeastern Mexico). The EPPC is a semi-circular structure, constituted by three emplacement centers, situated in the eastern part of the COIB and the northern extension of the Taxco-San Miguel de Allende fault system. However, the complex was not affected by the activity of this fault system. The EPPC is constituted by intrusive rocks varying from quartz monzodiorite to quartz syenite, the latter covering most of the complex surface. The EPPC was emplaced in Upper Cretaceous marine sedimentary rocks. The quartz syenite is cut by quartz monzodioritic and porphyritic meso-syenitic dykes. Also, this unit contains irregularly distributed monzodioritic microgranular enclaves. Additionally, pegmatitic dykes cut the rest of the lithologic
\end{abstract}


units. The EPPC mineralogical assemblage shows variable quantities of plagioclase + alkaline feldspar + quartz \pm amphibole + biotite \pm orthopyroxene + clinopyroxene $+\mathrm{Fe}$-Ti oxides. Intrusive rocks have a chemical composition of $\mathrm{SiO}_{2}=45.7-72.0 \%, \mathrm{Mg} \#=$ 39.2-60.2, and $n-F e=0.54-0.73$. They show chondrite-normalized REE patterns enriched in light elements $\left[(L a / Y b)_{N}=6-11\right]$ with no Eu anomalies. Primitive Mantle-normalized multi-element diagrams show LILE enriched patterns relative to the HFSE. Their trace-element geochemistry is comparable to high-Ba-Sr granitoids: (a) high Ba concentration (= 594-2302 ppm) and Sr (= 444-2192 ppm); (b) low concentrations of $Y(=10-46 \mathrm{ppm})$ and $\mathrm{Nb}(=6-17 \mathrm{ppm})$; and (c) high values for $\mathrm{Sr} / \mathrm{Y}(=25-85)$ and $(\mathrm{La} / \mathrm{Yb}=8.9-16.5)$ ratios. The origin of EPPC has been related to partial melting of an enriched lithospheric mantle, in a post-orogenic setting, followed by fractional crystallization coupled to crustal assimilation.

Keywords: Northeastern Mexico, high-Ba-Sr granitoids, post-orogenic magmatism.

\section{Introducción}

La historia geológica del Mesozoico al Terciario en el norte de México se caracteriza por el emplazamiento de un gran número de cuerpos intrusivos desde Baja California hasta Tamaulipas (e.g., Hoffer, 1970; Anderson y Silver, 1974; Clark et al., 1982; Nick, 1988; Morton-Bermea, 1990, 1995; Elías-Herrera et al., 1991; McDowell y Mauger, 1994; Stein et al., 1994; Ramírez-Fernández, 1996; González León et al., 2000; McDowell et al., 2001; Valencia-Moreno et al., 2001, 2003, 2006; Molina Garza et al., 2008; RamosVelázquez et al., 2008). Desde el Cretácico, principalmente, esta actividad magmática se ha relacionado con el complejo proceso de interacción entre el margen occidental de Norteamérica y la placa Farallón, que ha involucrado la generación de magmas de tipo arco continental (Coney y Reynolds, 1977; Clark et al., 1982; Damon et al., 1983; Humpreys et al., 2003). Adicionalmente, cambios en la velocidad de convergencia y del ángulo de subducción de la placa oceánica derivaron, durante el Mioceno, en condiciones de una tectónica distensiva que se reflejaron en el desarrollo de magmatismo intraplaca y fallamiento extensional sincrónico (Aranda-Gómez et al., 2005; Rangin et al., 2008).

De forma particular, entre Melchor Ocampo, Zacatecas, y el extremo occidental de Galeana, Nuevo León, se distribuye un grupo de complejos intrusivos caracterizados por un alineamiento general E-W (Figura 1) y que se han denominado colectivamente Cinturón de Intrusivos de Concepción del Oro (CICO; Chávez-Cabello et al., 2007). De acuerdo con la división propuesta para la Sierra Madre Oriental (SMOr) por Eguiluz de Antuñano et al. (2000), los intrusivos que constituyen el CICO se distribuyen entre los límites de la terminación oriental del Sector Transversal de Parras y el inicio de la Saliente de Monterrey (SM) en su extremo occidental (Servicio Geológico Mexicano, 2000a). Estudios areomagnéticos realizados en el CICO (Servicio Geológico Mexicano, 2000b) han revelado la existencia de anomalías magnéticas de gran magnitud y amplitud, las cuales se pueden asociar con los cuerpos intrusivos que afloran parcialmente. Estos cuerpos magmáticos están constituidos por troncos, diquestratos, diques, lacolitos y facolitos que, en general, cubren un intervalo de composición de diorita a sienita (Roger et al., 1957, 1963; Castro-Reino, 2004). Padilla y Sánchez (1982) asignó una edad relativa de emplazamiento del Oligoceno-Mioceno para el CICO, ya que algunos episodios de actividad magmática cortan estructuras laramídicas del Paleoceno-Eoceno (por ejemplo, los ejes de los pliegues de los anticlinales mayores). Castro-Reino (2004) reportó edades K-Ar terciarias para las rocas del CICO entre 35 y 40 Ma. Sin embargo, Mujica-Mondragón y Jacobo-Albarrán (1983) reportaron que los cuerpos sieníticos que afloran entre Coahuila, Nuevo León y Zacatecas (CICO) tienen edades K-Ar de $75 \pm 6$ Ma. Por otra parte, los intrusivos del CICO fueron emplazados en rocas sedimentarias de composición calcárea y margosa, las cuales se caracterizan por mostrar aureolas de metamorfismo de contacto pequeñas alrededor de los cuerpos intrusivos. Esta asociación ha dado lugar a mineralizaciones polimetálicas en forma de vetas, chimeneas y cuerpos irregulares, que incluyen metales base $(\mathrm{Pb}, \mathrm{Zn}, \mathrm{Cu}, \mathrm{Fe})$ y metales preciosos ( $\mathrm{Au} \mathrm{y} \mathrm{Ag})$, que han sido estudiados por varios autores (e.g., Chase, 1909; Krieger, 1940; Roger et al., 1957, 1963; De Cserna, 1976; COREMI, 1997; Castro-Reino, 2004).

Para incrementar el conocimiento acerca del magmatismo en el noreste de México, el presente estudio se enfocó en la caracterización mineralógica y geoquímica del Complejo Plutónico El Peñuelo o El Pedregoso (CPEP; Figura 1), un complejo plutónico situado en el extremo oriental del CICO. La información generada fue utilizada, considerando el marco geológico-estructural regional, para proponer un modelo conceptual inicial a fin de explicar el origen de este cuerpo intrusivo.

\section{Marco geológico-estructural}

El CPEP (Figuras 1 y 2 ) se encuentra parcialmente erosionado y se ubica geográficamente en el límite de los estados de Coahuila, Nuevo León, San Luis Potosí y Zacatecas, entre las coordenadas geográficas: 24³2'00" a $24^{\circ} 37^{\prime} 00^{\prime \prime} \mathrm{N}$ y $100^{\circ} 46^{\prime} 00^{\prime \prime}$ a $100^{\circ} 51^{\prime} 00^{\prime}$ ' W, cubriendo 


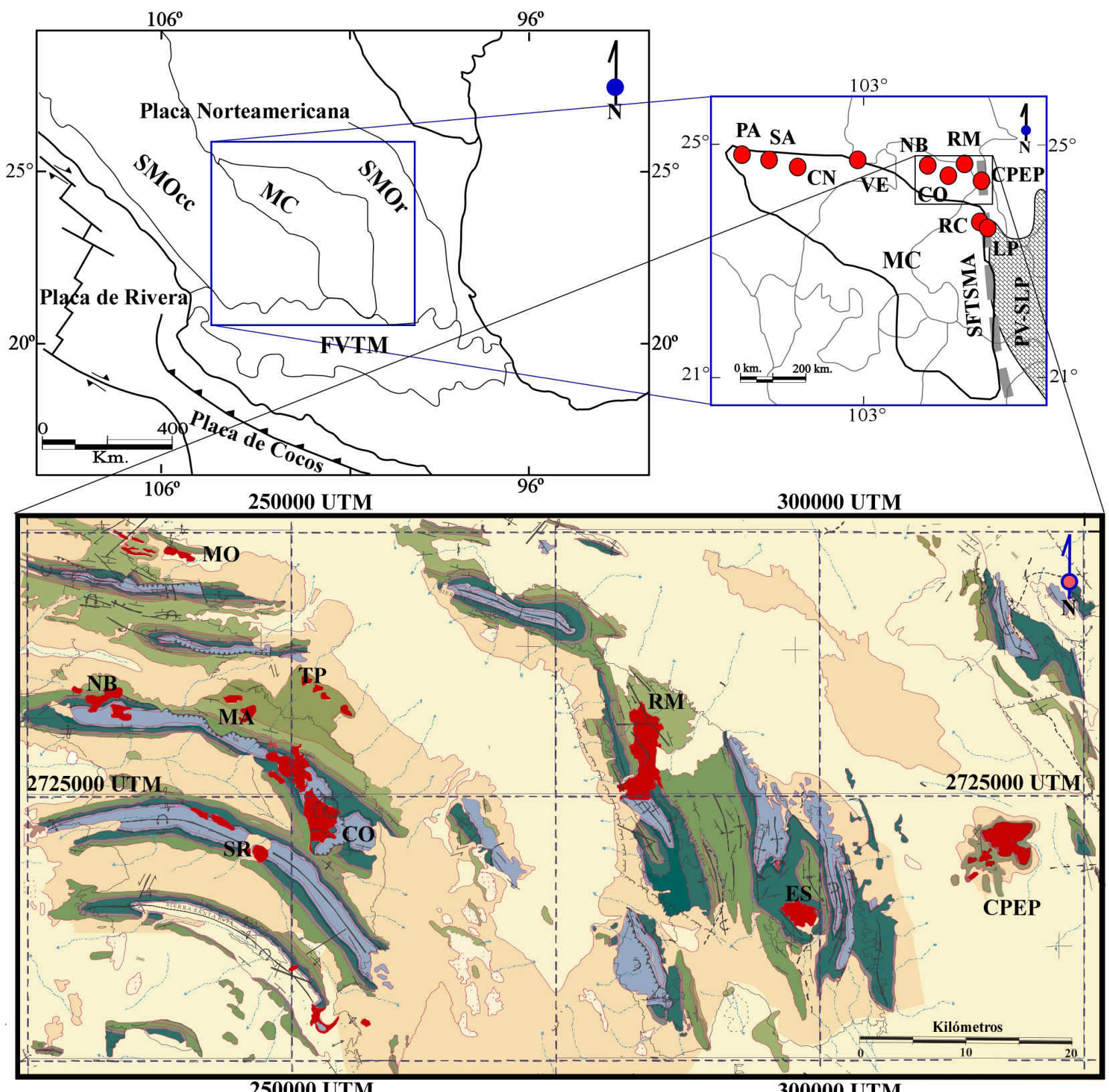

Figura 1. Localización regional y mapa geológico del Cinturón de Intrusivos de Concepción del Oro (CICO; modificado de Servicio Geológico Mexicano, 2000a). MC = Mesa Central; FVTM = Faja Volcánica Transmexicana; SMOcc $=$ Sierra Madre Occidental; SMOr $=$ Sierra Madre Oriental; PVSLP = Plataforma Valles-San Luis Potosí; SFTSMA = Sistema de Fallas Taxco - San Miguel Allende. Localidades de intrusivos de la zona norte de la MC: CN, Coneto de Comonfort; LP, La Paz; PA, Papantón; RC, Real de Catorce; SA: Sapiorís-América; VE, Velardeña. Localidades que conforman el CICO: CO, Concepción del Oro; CPEP, Complejo plutónico El Peñuelo; ES, El Saltillito; MA, Mazapil; MO, Melchor Ocampo; NB, Noche Buena; RM, Rocamontes; SR, Santa Rosa; TP: Terminal de Providencia.

un área de aproximadamente $60 \mathrm{~km}^{2}$. Se ubica en el extremo oriental del CICO y está situado además sobre la extensión al norte de la traza del sistema de Fallas Taxco-San Miguel de Allende (SFTSMA, orientado N-S; Figura 1). Cabe destacar que el SFTSMA separa una zona de plataformas continentales marinas de una amplia zona de mayor batimetría (Mar Mexicano; Alaniz-Álvarez et al., 2002). Esta discontinuidad paleogeográfica de edad cretácica controló la localización de fallamiento cenozoico de rumbo N-S y NNW-SSE y, anteriormente al fallamiento, el emplazamiento de cuerpos intrusivos con yacimientos minerales asociados, como Santa María de la Paz y Real de Catorce. A diferencia de la mayoría de las estructuras que conforman el CICO, el CPEP no muestra en superficie que corte estructuras laramídicas de la SMOr, aunque sus rocas encajonantes corresponden a rocas del Cretácico Superior (Formación Indidura; Turoniano inferior-Santoniano). Por otro lado, tampoco se observa que esté afectado por la 
$100^{\circ} 50^{\prime}$

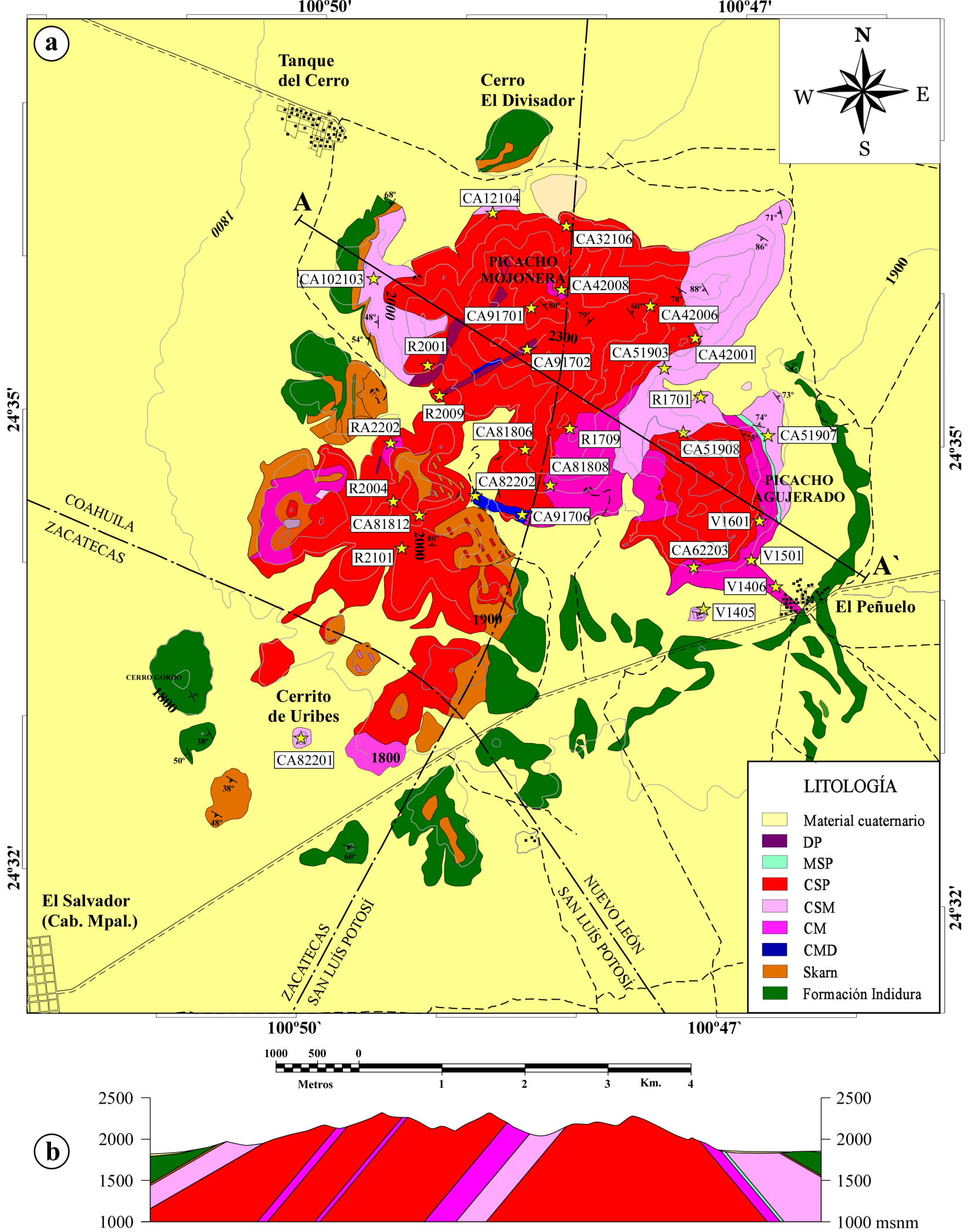

Figura 2. (a) Carta geológica generalizada del CPEP, mostrando la ubicación de los tres centros de emplazamiento del complejo (N, SW y SE); modificado de Chávez-Cabello et al. (2007). Litología: CMD, cuarzomonzodiorita; CM, cuarzomonzonita; CSP, cuarzosienita porfídica; CSM, cuarzosienita microcristalina; MSP, mesosienita porfídica; DP, diques aplíticos. La litología MD no está representada en la carta, ya que ocurre en forma de enclaves incluidos en CM y CS. Las estrellas indican la localización de los puntos de muestreo. (b) Perfil geológico A-A' con una orientación NW-SE y que corta el centro de emplazamiento $\mathrm{N}$ del CPEP. 
extensión relacionada al SFTSMA, el cual fue claramente activo entre San Miguel de Allende y Taxco a partir del Mioceno Medio (Pérez-Venzor et al., 1996; Alaniz-Álvarez et al., 2002), posiblemente debido a la gran distancia que existió ( $>1000 \mathrm{~km}$ ) entre el borde continental activo durante el Mioceno y el área de estudio.

El CPEP es una estructura semicircular, en planta, definida por tres centros de emplazamiento (Figura 2), los cuales han sido objeto de cartografía geológica, muestreo y análisis geoquímico. Presenta una serie de extensiones radiales, que podrían reflejar la misma disposición espacial de los conductos alimentadores. Estos estarían aproximadamente en concordancia con la orientación dominante del fracturamiento tensil regional, presente en las estructuras laramídicas que circundan y que podrían estar sepultadas en la zona. La información aeromagnética disponible para el área de estudio (Servicio Geológico Mexicano, 2000b), sugiere que las rocas máficas del complejo (mesosienitas porfídicas y cuarzo monzodioritas; índice de color $M^{\prime}=45-61$, Tabla 1, según LeMaitre et al., 2002), cuya exposición es limitada en superficie, así como alguna litología melanocrática que no aflora, serían las responsables de la respuesta magnética. Cabe señalar que la información detallada asociada a los principales elementos estructurales en el área de estudio y el modelo de emplazamiento de los plutones será presentado posteriormente.

\section{Litología y análisis petrográfico}

Se llevó a cabo un muestreo extensivo (aproximadamente $4 \mathrm{~kg}$ de muestra libre de alteración) en 29 sitios, el cual cubrió el espectro litológico del CPEP. Se realizó un análisis petrográfico, basado en la metodología propuesta para rocas graníticas de Melgarejo i Draper (1997), que incluye la determinación de tipo de textura, características particulares y abundancia de los diferentes minerales (conteo de 300 puntos/muestra, en un área aproximada de $4 \mathrm{~cm} \mathrm{x} 2.5 \mathrm{~cm}$ y con separación de $\sim 1 \mathrm{~mm}$ ). En la Tabla 1 y la Figura 3 se presentan, respectivamente, las características petrográficas distintivas de las unidades litológicas cartografiadas en el área de estudio y su clasificación de acuerdo al diagrama QAPF (Le Maitre et al., 2002).

Tabla 1. Localización e información petrográfica de las muestras colectadas en el CPEP.

\begin{tabular}{|c|c|c|c|c|c|c|c|c|c|c|c|c|c|c|c|c|c|c|}
\hline \multirow[b]{2}{*}{ Muestra } & \multirow[b]{2}{*}{ Localidad } & \multicolumn{2}{|c|}{ Coordenadas UTM } & \multicolumn{9}{|c|}{ Composición de minerales } & \multicolumn{6}{|c|}{ Clasificación petrográfica } \\
\hline & & Latitud (N) & Longitud $(\mathrm{W})$ & $Q z$ & $F A$ & $\mathrm{Plg}$ & $O p x$ & $C p x$ & Ttn & Anf & $B t$ & MO & Q & A & $P$ & $\mathrm{M}^{\prime}$ & Textura & Clase \\
\hline CA91701 & Picacho Mojonera & 721109 & 317435 & 1 & 25.6 & 11.9 & 8.2 & 12.6 & 9.9 & 9.9 & 8.9 & 11.9 & 2.6 & 66.5 & 30.9 & 61.4 & $\mathrm{P}$ & MSP \\
\hline R2101 & La Sanguijuela & 717746 & 315582 & 1.4 & 36.2 & 14 & 10 & 6.4 & 3.1 & 11.4 & 9.9 & 7.6 & 2.7 & 70.2 & 27.1 & 46.2 & $\mathrm{P}$ & MSP \\
\hline CA82202 & El Cercado & 718439 & 316196 & 2.6 & 11.3 & 25.6 & 3.6 & 10.3 & 4.4 & 14.3 & 16.6 & 11.3 & 6.6 & 28.6 & 64.8 & 60.3 & IG & CMD \\
\hline CA91706 & Manzanalea & 718153 & 316724 & 3 & 12.6 & 39.6 & 2.6 & 8.6 & 5.6 & 8.3 & 8.4 & 11.3 & 5.4 & 22.8 & 71.7 & 44.7 & IG & CMD \\
\hline V1406 & El Peñuelo & 717121 & 320882 & 1.5 & 12.1 & 37.5 & 11 & 12.9 & 3.7 & 7 & 2.6 & 11.8 & 2.9 & 23.7 & 73.4 & 48.9 & EG & MD \\
\hline V1501 & La Colmena & 717438 & 320656 & 1.9 & 13.7 & 36.3 & 10.7 & 12.2 & 5.2 & 5.6 & 1.5 & 13 & 3.7 & 26.4 & 69.9 & 48.1 & EG & MD \\
\hline CA62203 & La Colmena & 717432 & 319623 & 3.7 & 28.9 & 25.2 & 8.6 & 10.7 & 2.4 & 1.6 & 8.6 & 10.3 & 6.4 & 50 & 43.6 & 42.2 & IG & $\mathrm{CM}$ \\
\hline CA81808 & Mina El Zacate & 718621 & 317521 & 5 & 33.9 & 28.6 & 3.3 & 6 & 2.6 & 2 & 10.3 & 8.3 & 7.4 & 50.2 & 42.4 & 32.5 & IG & $\mathrm{CM}$ \\
\hline CA91702 & Picacho La Joya & 720864 & 317062 & 5 & 36.4 & 32 & 0.6 & 2.7 & 1.6 & 8.4 & 4 & 9.3 & 6.8 & 49.6 & 43.6 & 26.6 & IG & $\mathrm{CM}$ \\
\hline R2202 & Mina Daniel & 720172 & 314673 & 4.6 & 36.3 & 36.6 & 2 & 2.3 & 2 & 1 & 6 & 9.3 & 5.9 & 46.8 & 47.2 & 22.6 & IG & $\mathrm{CM}$ \\
\hline V1601 & Picacho Colorado & 718081 & 320057 & 5 & 27.6 & 36.3 & 1.3 & 4.6 & 6 & 1 & 10 & 8.2 & 7.3 & 40.1 & 52.7 & 47.1 & IG & $\mathrm{CM}$ \\
\hline CA12104 & Cañón El Pozo & 721984 & 316705 & 4.4 & 54.6 & 14.4 & 5 & 8.4 & 4.6 & - & 1 & 7.6 & 6 & 74.4 & 19.6 & 26.6 & $P$ & CSP \\
\hline CA32106 & Cañón Reta & 722347 & 317821 & 3.6 & 50.8 & 13.4 & 8.2 & 9.6 & 4.2 & - & 1 & 9.2 & 5.3 & 74.9 & 19.8 & 32.2 & $P$ & $\operatorname{CSP}$ \\
\hline CA42001 & Cerrito de Piedra & 722783 & 320867 & 4.8 & 45.2 & 22 & 1.4 & 5.2 & 5.2 & 1.4 & 6.5 & 8.3 & 6.7 & 62.8 & 30.6 & 27.9 & $\mathrm{P}$ & CSP \\
\hline CA81806 & Manzanales & 719176 & 317063 & 4 & 51 & 12 & 8.4 & 10 & 3 & 0.6 & 1 & 10 & 6 & 76.1 & 17.9 & 33 & $\mathrm{P}$ & $\operatorname{CSP}$ \\
\hline CA81812 & Puerto El Cercado & 718219 & 315705 & 4.3 & 42.5 & 16.1 & 3.1 & 13.5 & 3.7 & 11.1 & 2.4 & 2.3 & 6.8 & 67.6 & 25.6 & 36.1 & $\mathrm{P}$ & CSP \\
\hline R1709 & Los Tres Picachos & 719142 & 317876 & 4.3 & 52 & 17.2 & - & 10.7 & 2.2 & - & 2.2 & 11.4 & 5.9 & 70.7 & 23.4 & 26.5 & $\mathrm{P}$ & CSP \\
\hline R2001 & Manzanales & 720068 & 316435 & 4.7 & 44.4 & 16.5 & 3.4 & 8.6 & 3 & 9.6 & 8.8 & 1 & 7.2 & 67.7 & 25.2 & 34.3 & P & CSP \\
\hline R2004 & Cañón La Alberca & 718562 & 315347 & 4 & 46 & 18.3 & 3.3 & 4 & 4.6 & 10.3 & 3.2 & 6.3 & 5.9 & 67.3 & 26.8 & 31.7 & $\mathrm{P}$ & CSP \\
\hline V1405 & Cerro Peñuelos & 716889 & 319784 & 4.6 & 46.6 & 13.2 & 3 & 9.1 & 4.3 & 2.2 & 5.6 & 10.4 & 7.1 & 72.4 & 20.5 & 34.6 & $\mathrm{P}$ & CSP \\
\hline CA51903 & La Yerbabuena & 720413 & 318892 & 5.6 & 45.6 & 14.6 & 5 & 5 & 1.6 & 8.6 & 5.2 & 8.8 & 8.5 & 69.3 & 22.2 & 34.2 & IG & CSM \\
\hline CA51907 & Picacho Agujereado & 719346 & 320387 & 6.7 & 51.5 & 18 & 2.2 & 4.6 & 2.6 & 1.6 & 3.3 & 9.5 & 8.8 & 67.6 & 23.6 & 23.8 & IG & CSM \\
\hline CA51908 & Picacho Agujereado & 719324 & 319380 & 6 & 48 & 15.6 & 1.6 & 5 & 8.6 & 2 & 5 & 8.2 & 8.6 & 69 & 22.4 & 30.4 & IG & CSM \\
\hline CA102103 & Cañón El Zopilote & 721145 & 315117 & 6.1 & 49.5 & 12.6 & 0.6 & 4.4 & 4.8 & 0.6 & 7.4 & 14 & 8.9 & 72.6 & 18.5 & 31.8 & IG & CSM \\
\hline R1701 & La Yerbabuena & 719925 & 319712 & 7.3 & 53.7 & 17.8 & 1 & 3.8 & 5.7 & - & 3.6 & 7 & 9.3 & 68.1 & 22.6 & 21.1 & IG & CSM \\
\hline CA82201 & Cerrito de Uribes & 715051 & 314039 & 14.3 & 71.7 & 4.7 & 2.7 & 2 & 1.3 & 0.3 & 0.3 & 2.7 & 15.8 & 79.1 & 5.2 & 9.3 & $\mathrm{FE}$ & DP \\
\hline R2009 & Cañón La Pastora & 719786 & 315760 & 13.7 & 73 & 4.3 & 3 & 1 & 1.3 & 1 & 0.7 & 2 & 15.1 & 80.2 & 4.7 & 9 & $\mathrm{FE}$ & DP \\
\hline
\end{tabular}

Composición modal, basada en el análisis de $~ 300$ puntos/lámina, y clasificación petrográfica de acuerdo al esquema QAPM' y la textura (Le Maitre et al., 2002). Abreviaturas: (a) Minerales: $\mathrm{Qz}=$ cuarzo, FA = feldespato alcalino, Plg $=$ plagioclasa, Opx $=$ ortopiroxeno, Cpx $=$ clinopiroxeno, Ttn $=$ titanita, Anf = anfíbol, Bt = biotita, $\mathrm{MO}=$ minerales opacos; (b) Clasificación QAPM': Q = cuarzo, $\mathrm{A}=$ feldespato alcalino, $\mathrm{P}=$ plagioclasa, $\mathrm{M}^{\prime}=$ índice de color; (c) Textura: $\mathrm{P}=$ porfídica, $\mathrm{IG}=$ inequigranular, $\mathrm{EG}=$ equigranular, $\mathrm{FE}=$ fanerítica equigranular; $(\mathrm{d}) \mathrm{Clase}$ : $\mathrm{MSP}=$ mesosienita porfídica, $\mathrm{CMD}$ $=$ cuarzomonzodiorita, $\mathrm{MD}=$ monzodiorita, $\mathrm{CM}=$ cuarzomonzonita, $\mathrm{CSP}=$ cuarzosienita porfídica, $\mathrm{CSM}=$ cuarzosienita microcristalina, $\mathrm{DP}=$ dique pegmatítico. 


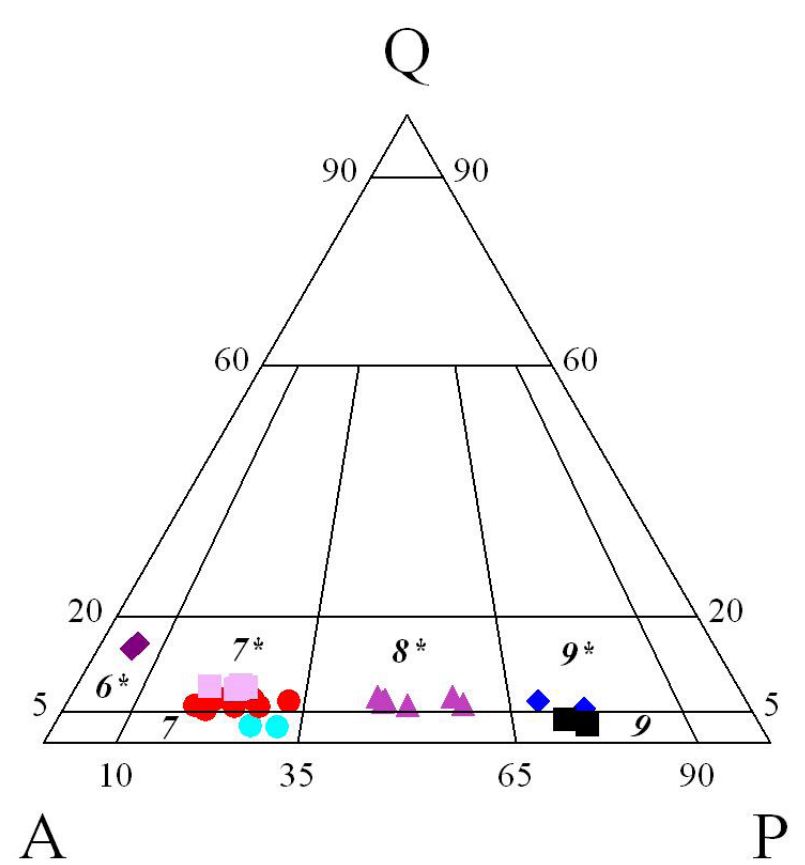

Figura 3. Figura 3. Diagrama QAP, (LeMaitre et al., 2002), que muestra la clasificación modal para las muestras colectadas en el CPEP. Campos: $6^{*}=$ cuarzosienita de feldespato alcalino $7=$ sienita; $7^{*}=$ cuarzosienita; $8^{*}=$ cuarzomonzonita; $9=$ monzodiorita; $9^{*}=$ cuarzomonzodiorita La clasificación del campo de la cuarzosienita se complementa con el parámetro $M^{\prime}$ (índice de color) y la textura macroscópica. Los enclaves autolíticos, presentes en la CSP, se ubican en el campo de la monzodiorita (campo \# 9). Para distribución de las muestras en el diagrama, consultar la Figura 2 y la Tabla 1.

\subsection{Roca encajonante}

Los estratos calcáreo-arcillosos de la Formación Indidura (Cenomaniano-Turoniano; Duque-Botero y Maurrasse, 2008) constituyen la roca encajonante que aflora alrededor del CPEP. La unidad está constituida por calizas arcillosas de tipo mudstone-wackstone de color gris obscuro que intemperiza a color rojo, cuyas capas varían entre $10 \mathrm{y}$ $40 \mathrm{~cm}$ de espesor, intercaladas con estratos de 30 a $70 \mathrm{~cm}$ de lutitas y limolitas calcáreas. El espesor total de la unidad en el área de estudio no se determinó, debido a que la base y el techo no afloran en su totalidad. La Formación Indidura se ha interpretado como un depósito de sedimentos en un medio batial a sublitoral de cuenca en donde prevalecieron condiciones reductoras, confirmadas por la presencia de nódulos de fierro y la acumulación de materia orgánica (Kelly, 1936; Jones, 1938; Tristán-González y TorresHernández, 1994; Duque-Botero y Maurrasse, 2008).

En el área de estudio, la Formación Indidura presenta una deformación asociada al emplazamiento magmático, como lo evidencian algunos pliegues con longitudes de onda de escala de decenas de centímetros y el desarrollo de foliaciones tectónicas paralelas al contacto plutón-roca encajonante. En los bordes de los sectores $\mathrm{N}$ y principalmente SW del intrusivo, se reconoció una aureola de contacto, que se presenta principalmente en forma de endoskarn (Figura 2; color café). El endoskarn se caracteriza por mostrar una textura poiquilítica, que incluye fenocristales de feldespatos alcalinos, plagioclasas, clinopiroxenos, granates, anfíboles, wollastonita y remanentes de calcita (Figura 5A). En otras localidades, el exoskarn está presente y se encuentra cortado por intrusiones sieníticas y diques aplíticos de espesores $<$ $6 \mathrm{~cm}$ (Figura 4A), aunque también puede encontrarse en forma de xenolitos dentro del cuerpo intrusivo.

\subsection{Litología del cuerpo intrusivo}

Considerando relaciones de campo, constituyentes minerales y rasgos texturales, el CPEP se encuentra integrado por los siguientes tipos litológicos (Figura 3), en orden de menor a mayor contenido de sílice: (1) mesosienita porfídica (MSP); (2) cuarzomonzodiorita (CMD); (3) cuarzomonzonita (CM); (4) cuarzosienita (CS), con dos variedades: cuarzosienita porfídica (CSP) y cuarzosienita microcristalina (CSM); (5) diques pegmatíticos (DP); y (6) autolitos microdioríticos (MD), presentes en la CM y CS. La CMD representa la unidad ígnea más antigua y la CS es la más abundante (Figura 2). Las características distintivas de cada unidad litológica, observadas en campo y en lámina delgada, se describen en los siguientes apartados siguiendo el criterio de menor a mayor contenido de sílice.

\subsubsection{Meso-sienita porfidica (MSP)}

Ocurre en forma de diques, cuyos espesores varían de 1 hasta máximo $40 \mathrm{~m}$, expuestos en los sectores N y SE, únicamente en la parte $S E$ el cuerpo podía ser cartografiable por su espesor (Figura 2; color celeste). Esta unidad ígnea incluye una gran cantidad de fenocristales euedrales de ortoclasa ( $\sim 5-15 \mathrm{~cm}$ de diámetro) alineados, embebidos en una matriz máfica microcristalina o afanítica (Figura 4B). Se infiere una interacción física con la CSP ya que, dentro de la MSP, los cristales de ortoclasa presentan bordes de desequilibrio con la matriz que los contiene.

El estudio petrográfico de esta unidad reveló una textura porfídica holocristalina (Figura 5B). Su característica petrográfica distintiva es la presencia de fenocristales de ortoclasa euedral (25-37\%) de 0.5 a $1.5 \mathrm{~mm}$ de longitud, dentro de los cuales pueden ocurrir inclusiones minerales de plagioclasa y feldespato potásico de 0.1 a $0.5 \mathrm{~mm}$ de largo. La plagioclasa (11-14\%), cuyo diámetro varía entre 0.5 y $1.2 \mathrm{~mm}$, exhibe formas euedrales, con maclado polisintético y de Carlsbad, así como subedrales, caracterizadas por albitización. El resto de la mineralogía está compuesta por anfíboles anedrales $(9-12 \%)$ de 0.3 a $1.0 \mathrm{~mm}$ de diámetro, biotita subedral (8-10\%) de 0.3 a $2.4 \mathrm{~mm}$ de diámetro máximo, ortopiroxenos subedrales $(8-10 \%)$ de 0.3 a 1.0 $\mathrm{mm}$ de longitud, clinopiroxenos subedrales (6-13\%) de 0.3 a $0.7 \mathrm{~mm}$ de longitud, y titanita euedral (3-10\%) de 0.2-0.7 $\mathrm{mm}$ de diámetro. Este arreglo mineralógico se complementa con acumulaciones de microcristales de cuarzo $(<2 \%,<0.5$ $\mathrm{mm}$ de diámetro) y minerales opacos diseminados (7-12\%). 


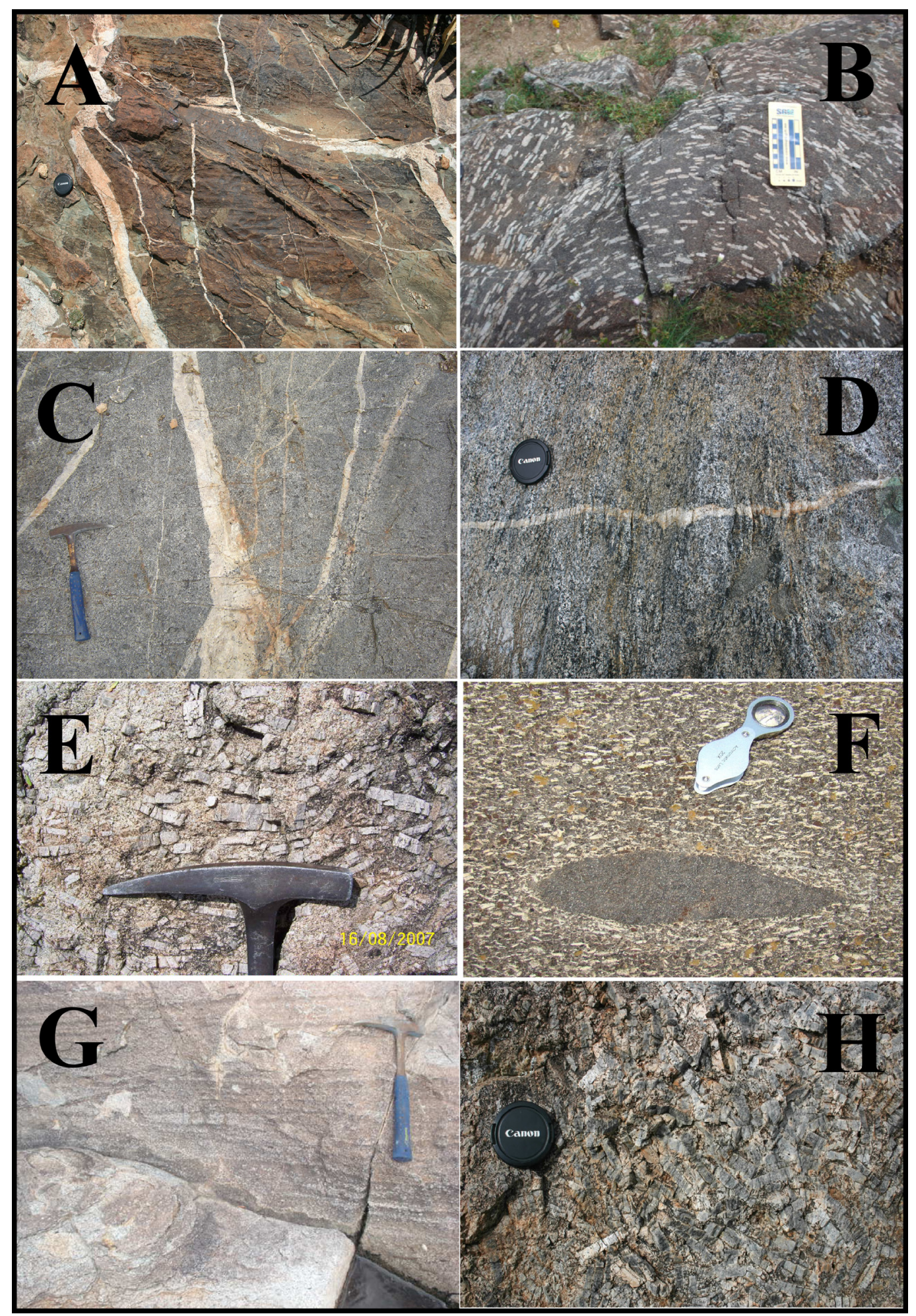

Figura 4. Imágenes de campo de las litologías que ocurren en el CPEP: (A) Roca intrusiva metamorfizada (endoskarn) de color rojizo en el borde SW del intrusivo, que incluyen dos familias de vetas aplíticas (localidad: cañada El Zacate); (B) Dique de MSP, donde se observa un cambio de rumbo de la dirección en la lineación magmática, según los pórfidos de ortoclasa euedrales (localidad: picacho Mojonera); (C) Afloramiento de CMD, con familia de vetas aplíticas que muestran una orientación $\sim \mathrm{N}-\mathrm{S}$ (localidad: cañada El Cercado); (D) Afloramiento de CM en su transición a LCSP, en donde se aprecian autolitos microgranitoides, foliación y una veta horizontal aplítica (localidad: cañada La Escondida); (E) Afloramiento de CSP, donde se observa una alineación magmática con rumbo aproximado SW-NE, de acuerdo a fenocristales de ortoclasa euedral (localidad: cañón Reta); (F) Autolito microgranitoide (MD) de forma lenticular embebido en la CSP, en donde se aprecian bordes de enfriamiento producto de mingling (localidad: El Peñuelo); (G) Afloramiento de CSM en donde se aprecia la dirección de la foliación composicional de tipo bandeada (N-S) (localidad: cañón El Zopilote); (H) Dique pegmatítico, conformado principalmente por cristales euhédricos de feldespato alcalino (localidad: cañón El Zopilote). Escalas en A, D y H: diámetro de tapa para lente de cámara $=8 \mathrm{~cm}$; en B: escala vertical en tarjeta $=10 \mathrm{~cm}$; en C, E y G: longitud horizontal de martillo geológico $=17 \mathrm{~cm}$; en F: diámetro de lupa $=15 \mathrm{~mm}$. 


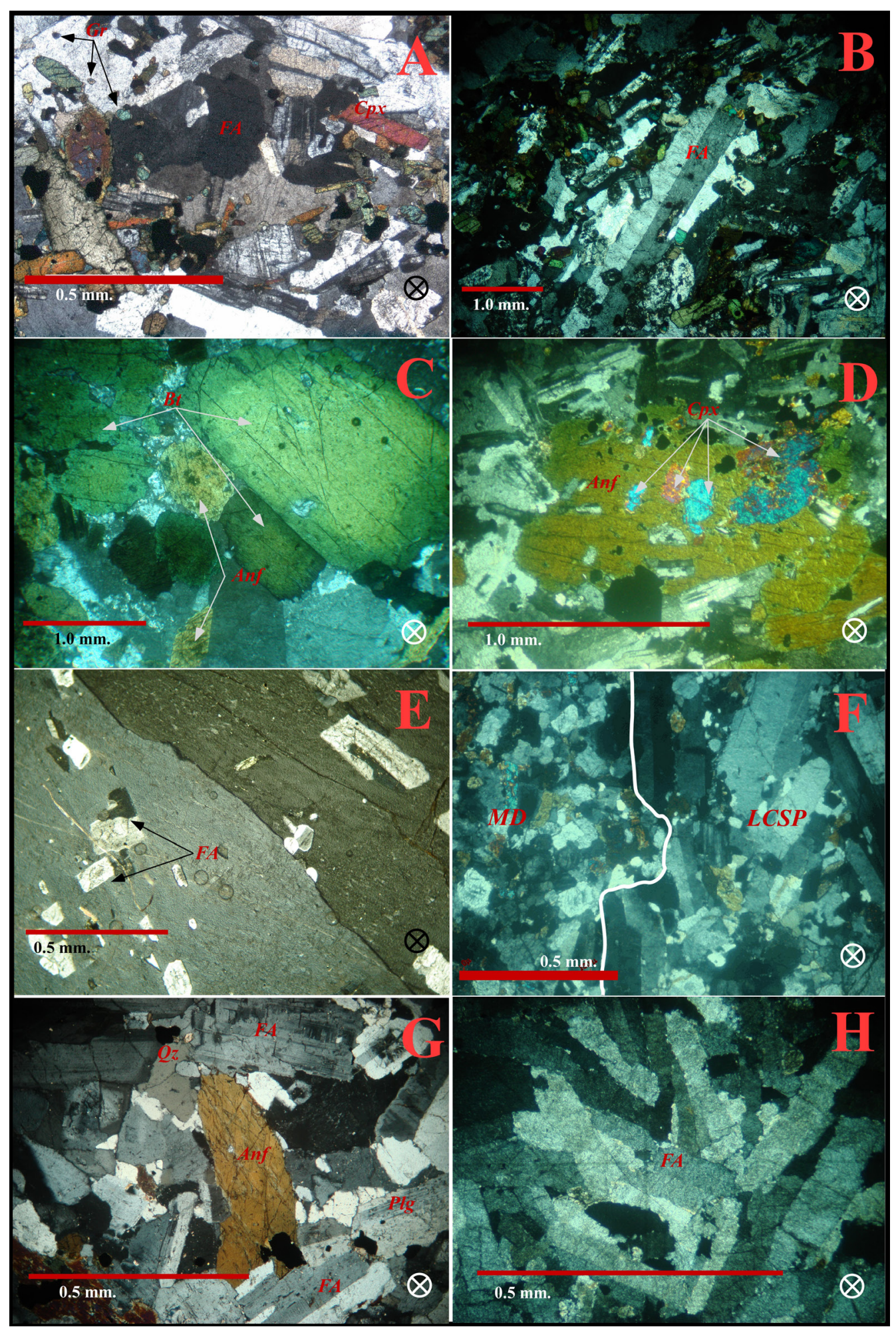

Figura 5. Fotomicrografías de láminas delgadas (nícoles cruzados, U) para las litologías que ocurren en el CIEP: (A) Endoskarn de textura poiquilítica (localidad: Cañada El Zacate); (B) Dique de MSP, en donde se observa un fenocristal de ortoclasa euedral intrafracturado con bordes irregulares, en contacto principalmente con minerales máficos (R2101; localidad: La Sanguijuela); (C) Litología CMD de textura inequigranular holocristalina, en donde se aprecian biotita y anfíbol (CA82202; localidad: El Cercado); (D) Cristal de anfíbol en una CM, el cual presenta inter-crecimiento de clinopiroxeno, que le confiere una textura de criba (V1601; localidad: Picacho Colorado); (E) Inter-crecimiento de feldespato alcalino dentro de un fenocristal de ortoclasa intra-fracturado, incluidos en una CSP (CA32106; localidad: cañón Reta); (F) Contacto de MD (autolito microgranitoide) con CSP, constituidos principalmente por feldespatos alcalinos orientados en ambas rocas (V1405; localidad: Cerro Peñuelos) (G) Arreglo de anfíbol subedral y cuarzo, rodeados por feldespato alcalino, presentes en una CSM (CA102103; localidad: cañón El Zopilote); (H) Imagen de un dique pegmatítico equigranular, constituido por feldespato alcalino y cuarzo (CA82201; localidad: Cerrito de Uribes). Anf = anfíboles; $\mathrm{Bt}=$ biotita; $\mathrm{Cpx}=$ clinopiroxenos; $\mathrm{FA}=$ feldespatos alcalinos, $\mathrm{Plg}=$ plagioclasas, $\mathrm{Q}=$ cuarzo . 


\subsubsection{Cuarzomonzodiorita (CMD)}

Aflora en el complejo $\mathrm{N}$ en forma de cuerpos tabulares de $800 \mathrm{~m} \times 200 \mathrm{~m}$ y $400 \mathrm{~m} \times 70 \mathrm{~m}$, algunos de ellos paralelos al perímetro de CSP y de aspecto radial (Figura 2; color azul). La CMD exhibe una textura fanerítica y tonalidad gris en muestra fresca. Se presenta generalmente como un cuerpo sumamente erosionado entre los límites de los sectores $\mathrm{N}$ y SW del CPEP. Generalmente se observa intrusionada por una gran cantidad de diques pegmatíticos y vetas aplíticas (Figura 4C). Adicionalmente, esta unidad litológica ocurre, al $\mathrm{E}$ del sector $\mathrm{SW}$ y al $\mathrm{SW}$ del sector $\mathrm{N}$, de forma esporádica $\mathrm{y}$ en afloramientos no representables a la escala del mapa. Los minerales que componen la CMD son de grano medio $\mathrm{y}$ contienen formas irregulares que no sobrepasan $\operatorname{los} 2 \mathrm{~cm}$. Sin embargo, se han identificado agregados de minerales máficos, principalmente anfíboles y piroxenos, formando texturas cumulíticas. A diferencia de la MSP, en esta unidad no se observa una orientación preferencial para los cristales.

$\mathrm{El}$ análisis petrográfico (Figura 5C) revela una textura inequigranular holocristalina localmente poiquilítica, conformada por plagioclasa, feldespato alcalino, anfíbol y biotita. La plagioclasa (25-40 \%) se presenta en forma euedral (0.5-2.0 $\mathrm{mm}$ de diámetro) y exhibe maclado polisintético y de Carlsbad. El feldespato alcalino (11-13 $\%$ ) se presenta en forma de cristales euedrales a subedrales de 0.8-2.0 $\mathrm{mm}$ de diámetro. Los anfíboles (8-15\%) se presentan como cristales subedrales a anedrales de 0.5-2.4 $\mathrm{mm}$ de longitud, con pleocroísmo intenso de color café. La biotita (8-17\%) ocurre en forma euedral de 0.5 a $3.0 \mathrm{~mm}$ de diámetro máximo. La mineralogía de la roca se complementa con minerales opacos (11-12\%), ortopiroxenos (8-11\%), clinopiroxenos $(<4 \%)$ y cuarzo $(<3 \%)$. La titanita $(<6$ $\%, 0.4-0.7 \mathrm{~mm}$ de diámetro) es accesoria en esta unidad.

\subsubsection{Cuarzomonzonita (CM)}

Se presenta como una unidad de grano medio con tonalidades verde-grisáceas en muestra fresca (Figura 2, en color magenta, y Figura 4D). Aflora principalmente en la parte centro y $\mathrm{S}$ del complejo intrusivo, rodea a los sectores SW y SE, también aparece en la parte centro y $\mathrm{S}$ del sector $\mathrm{N}$, generalmente en niveles topográficos bajos formando una estructura cercanamente anular con diques radiales. Los contactos litológicos son intrusivos tipo corte de cuchillo con zonas de brechas magmáticas $(\sim 0.5-20 \mathrm{~m})$, debido al fracturamiento de la roca encajonante durante el emplazamiento magmático. Los minerales constituyentes principales, en orden de abundancia: plagioclasas, anfíboles y piroxenos, que con frecuencia muestran orientación preferencial cerca y en el contacto de la CM con las rocas que la encajonan (Figura 4D).

$\mathrm{La} \mathrm{CM}$ se caracteriza por una textura inequigranular holocristalina (Figura 5D), constituida principalmente por plagioclasa subedral (25-40\%, 0.3-2.0 mm de diámetro), con zonación oscilatoria mostrando maclas polisintéticas y de Carlsbad, el feldespato alcalino presente (25-41\%,
0.3-4.1 mm de diámetro) ocasionalmente presenta texturas pertíticas. La biotita (4-11\%) exhibe formas principalmente euedrales de 0.5 a $1.8 \mathrm{~mm}$ de diámetro máximo, un hábito hojoso, e inclusiones de minerales máficos. El anfíbol (1-8 \%) se presenta en forma de cristales $(0.5-2.4 \mathrm{~mm}$ de longitud) subedrales y anedrales de color café, con inclusiones de minerales máficos dentro de los cristales de mayor tamaño, confiriéndole una textura tipo poiquilítica. El cuarzo (3-5\%) se presenta como granos subedrales de 0.3$0.5 \mathrm{~mm}$ o en formas anedrales de $0.5-1.0 \mathrm{~mm}$ de diámetro, con extinción ondulante y desarrollo de pequeñas fracturas. La mineralogía se complementa con ortopiroxenos (0.5-11 $\%)$ y clinopiroxenos (1-9 \%) de 0.2-1.0 mm de longitud, titanita euedral ( $1-7 \%, 0.2-0.7 \mathrm{~mm}$ de diámetro) y minerales opacos diseminados.

\subsubsection{Cuarzosienita (CS)}

Es la unidad más abundante en superficie, aflorando en los tres centros de emplazamiento. Se presenta en dos variedades: (a) cuarzosienita porfídica (CSP; Figura 2, color rojo), y (b) cuarzosienita microcristalina (CSM; Figura 2, color rosa). En algunas localidades, los cristales constituyentes de la roca muestran una orientación preferencial, principalmente en los bordes del sector $\mathrm{N}$, cerca del contacto con la roca encajonante. La CSP (Figura 4E) exhibe tonalidades grises en muestra fresca. Su característica principal es la ocurrencia de fenocristales euedrales de ortoclasa. En muestra de mano se pueden distinguir además piroxenos y hornblenda $(<2 \mathrm{~cm})$.

La textura dominante de la CS es porfídica holocristalina (Figura 5E), con una predominancia de feldespatos alcalinos (42-55\%, 0.5-1.5 mm de diámetro), principalmente como ortoclasa, y esporádicamente microclina con texturas pertíticas. La plagioclasa (13-22 \%, 0.5-1.0 mm de diámetro) es principalmente euedral con maclado polisintético y de Carlsbad, y también presenta formas subedrales con zonaciones oscilatorias. El cuarzo (3-5\%) ocurre como pequeños granos subedrales de $0.2-0.5 \mathrm{~mm}$ de diámetro, con cristales de $1.0 \mathrm{~mm}$ rellenando espacios entre los minerales. La hornblenda (9-11 \%) se presenta de formas subedrales y euedrales, de color café y con longitudes de $0.3-0.8$ $\mathrm{mm}$. La biotita (1-9 \%, 0.3-0.8 $\mathrm{mm}$ de diámetro máximo) exhibe una forma euedral con hábito hojoso y pleocroísmo intenso. La titanita (3-5 \%) se presenta de forma euedral romboidal, con tamaños de 0.2 a $0.5 \mathrm{~mm}$. La mineralogía restante consiste en ortopiroxenos (5-11\%, 0.1-1.0 mm de longitud), clinopiroxenos (4-11\%, 0.2-1.0 mm de longitud) y pequeños cristales diseminados de minerales opacos con alto magnetismo (7-12\%, 0.1-0.5 mm de diámetro) .

De forma esporádica, principalmente en los sectores $\mathrm{Ny}$ $\mathrm{SE}$, la CSP contiene enclaves máficos microgranitoides $(<50$ $\mathrm{cm}$; Figura 4F), de forma lenticular y paralelos a la foliación magmática. Algunos de estos enclaves microgranitoides también se encuentran en la CM. En el diagrama QAPF (Figura 3; Tabla 1), los enclaves máficos microgranitoides se localizan en el campo de la monzodiorita (MD). Se 
caracterizan por una textura equigranular holocristalina, con una clara disminución en el tamaño de grano en comparación con la roca que los contiene (Figura 5F). Su mineralogía está dominada por cristales subedrales de plagioclasas (36-38 $\%)$, que muestran un maclado polisintético y de Carlsbad (0.2-0.5 mm de diámetro), feldespatos alcalinos (12-14\%), acompañado generalmente por cristales de cuarzo que los rodean $(<2 \%,<0.3 \mathrm{~mm}$ de diámetro). Los enclaves máficos microgranitoides también incluyen piroxenos $(22-24 \%$, 0.2-0.3 mm de longitud), hornblenda (5-7 \%, 0.1-05 mm de longitud) con bordes alterados a clorita, y biotita euedral (1-3 \%, 0.1-0.3 mm de diámetro máximo). La mineralogía se complementa con pequeños cristales de minerales opacos (óxidos de Fe-Ti) dispersos dentro de los enclaves máficos (11-13\%, 0.1 mm de diámetro).

La CSM presenta una textura inequigranular de grano fino, color gris claro en muestra fresca, que intemperiza a tonalidades ocre y amarillentas. Generalmente los afloramientos muestran un aspecto bandeado (Figura $4 \mathrm{G})$. Aflora principalmente en el borde de los centros de emplazamiento en las porciones N y SE. En esta unidad litológica se puede distinguir ortoclasa, plagioclasas y hornblenda. Además, en algunos afloramientos la roca exhibe bandas de ortoclasa paralelas a la foliación.

El estudio petrográfico de la CSM (Figura 5G) revela una textura inequigranular holocristalina conformada de cristales euedrales a subedrales de feldespatos alcalinos (45-54\%, 0.3-1.2 mm de diámetro), predominando la ortoclasa sobre la microclina (0.3-0.5 mm de diámetro). Las plagioclasas se presentan como cristales euedrales tabulares con maclado polisintético y de Carlsbad, o como fenocristales subedrales, caracterizada por un zonado oscilatorio (12-18\%, 0.3$1.2 \mathrm{~mm}$ de diámetro). El cuarzo se presenta en cristales anedrales (5-8 \%, 0.2-0.5 mm de diámetro), con extinción ondulante. La hornblenda se presenta en cristales subedrales a anedrales de color café ( $<2 \%, 0.3-1.0 \mathrm{~mm}$ de diámetro); en una muestra los anfíboles constituyen hasta el $9 \%$ de la roca. La biotita exhibe formas euedrales (3-8 \%, 0.3-0.8 $\mathrm{mm}$ de diámetro máximo). La titanita se presenta de forma euedral romboidal (1.9\%, 0.2-0.5 mm de diámetro). Los ortopiroxenos exhiben tonalidades amarillas a verdes (3-5 $\%, 0.3-0.5 \mathrm{~mm}$ de longitud), mientras que los clinopiroxenos muestra tonos verdes pálidos a incoloros $(0.5-5 \%, 0.3-1.0$ $\mathrm{mm}$ de longitud). Son comunes los minerales opacos (7-14 $\%, 0.1-0.5 \mathrm{~mm}$ de diámetro).

\subsubsection{Diques pegmatíticos (DP)}

Existe un número importante de diques pegmatíticos (Figura 2, color morado, y Figura 4H) emplazados tardíamente dentro del CPEP. Estos diques a su vez están cortados por vetillas de composición aplítica, presentes a través de todo el complejo. De acuerdo con las observaciones de campo, se puede inferir que los diques aprovecharon el fracturamiento extensional preexistente en la zona, lo cual se puede ver más claramente en el Cerrito de Uribes, una estructura ubicada al S del sector SW del CPEP.
Los DP presentan una textura fanerítica equigranular (0.1-0.3 mm de diámetro), dominada por feldespatos alcalinos (71-73\%) en formas euedrales con maclado de Carlsbad (Figura 5H). El segundo mineral en importancia es el cuarzo (13-15\%), presentándose como cristales intersticiales. Las plagioclasas (4-5\%) se presentan en formas tabulares, con un maclado polisintético o de Carlsbad. La mineralogía se complementa con cantidades menores de ortopiroxenos, clinopiroxenos, anfíboles, biotita y titanita $(<3 \%)$. Pueden apreciarse también pequeñas cantidades de minerales opacos (óxidos de Fe, 2-3\%).

\section{Geoquímica}

\subsection{Geoquímica analítica}

Una vez establecidas las características petrográficas, se seleccionaron 18 muestras para determinar su composición en elementos mayores y traza aplicando métodos instrumentales. Para los análisis químicos, $\sim 0.5 \mathrm{~kg}$ de cada muestra fueron triturados hasta obtener fragmentos de 0.5 $1.0 \mathrm{~cm}^{3}$. El tamaño de los fragmentos se redujo a $<0.5 \mathrm{~mm}^{3}$ en un triturador de tipo Siebtechnik EB 7/6 y, finalmente, cada muestra fue pulverizada a un tamaño de partícula de 33-75 $\mu \mathrm{m}$ en un molino de ágata de tipo Siebtechnik Ts 100.

Adicionalmente, se realizó la separación de un enclave de MD, desde la muestra V1406. Para este fin se utilizó una cortadora con disco diamantado y se retiró la matriz de la roca que lo contenía, hasta dejarlo libre de impurezas, obteniendo una muestra de $\sim 8 \times 3 \mathrm{~cm}$.

La composición de elementos mayores y traza se determinó en los laboratorios comerciales ActLabsSkyline (Ancaster, Canadá), aplicando la metodología 4LithoResearch. La determinación de elementos mayores consistió en mezclar la muestra con un fundente "met-tet" (metaborato - tretraborato de litio) en una relación 1:4. La mezcla resultante fue fundida en un horno de inducción $\mathrm{a} \sim 1100^{\circ} \mathrm{C}$. El líquido de fusión se mezcló en caliente con una solución de $\mathrm{HNO}_{3} 5 \%$ ultra-puro y una serie de estándares internos, hasta disolución completa ( 30 min). La solución resultante fue analizada en un sistema Thermo Jarrell-Ash ENVIRO II ICP-OES, el cual fue previamente calibrado utilizando una combinación de materiales de referencia geoquímica (U.S. Geological Survey: dolerita DNC-1, diabasa W-1, basalto BIR-1, jasperoide GXR-1, sedimento GRX-2; Geological Survey of Canada: gabro WMG-1; British Geological Survey: sedimento LKSD3; Geological Survey of Poland: apatita CTA-AC-1). En general, la precisión analítica reportada fue $<2 \%$ para todos los elementos mayores, mientras que los límites de detección estuvieron en niveles de $0.01 \%$.

La composición en elementos traza, incluyendo tierras raras, se determinó aplicando, en primera instancia, un proceso de fusión y disolución similar al utilizado para elementos mayores. A la solución resultante se le añadió 
un estándar interno, que incluyó los elementos a analizar. Posteriormente, la muestra fue diluida con agua ultrapura y se introdujo en un sistema Perkin-Elmer SCIEX ELAN 6000 $I C P-M S$. Al igual que en el análisis de elementos mayores, el sistema analítico ICP-MS fue calibrado aplicando una serie de materiales de referencia geoquímica. La precisión analítica para la mayoría de los elementos analizados fue de $5-12 \%$, mientras que los límites de detección variaron entre 0.5 y $10 \mathrm{ppm}$.

\subsection{Características geoquímicas de las rocas intrusivas}

La composición geoquímica de las muestras analizadas se presenta en las Tablas 2 y 3 . Esta información fue compilada en el paquete comercial STATISTICA for MS WINDOWS 7.0 (Statsoft, Inc., Tulsa, Oklahoma, 19842004). Los datos de elementos mayores fueron procesados utilizando el programa SINCLAS (Verma et al., 2002, 2003) para normalizar los datos a una base seca, así como para calcular la norma $C I P W$ (Wilson, 1989; Rollinson, 1993).

De acuerdo con la clasificación de Frost et al. (2001), con la excepción de MSP y una CM, las rocas del CPEP presentan características comparables a las de los granitoides cordilleranos (Figura $6 \mathrm{~A}$ ), con una relación $\mathrm{FeO} /(\mathrm{FeO}+$ $\mathrm{MgO}$ ) < 0.7. Según el parámetro MALI (Modified Alkali Lime Index), basado en la relación $\left[\mathrm{Na}_{2} \mathrm{O}+\mathrm{K}_{2} \mathrm{O}-\mathrm{CaO}\right]$ (Figura 6B), éstas muestran un carácter cálcico a alcalino. Las litologías MSP, MD (con excepción de V1406), CMD y CM muestran nefelina y olivino normativos, mientras que las rocas incluidas en los grupos CSP, CSM y DP son normativas en cuarzo (Tabla 2). De acuerdo a sus relaciones molares de $\mathrm{Al} /[\mathrm{Ca}-1.67 \mathrm{P}+\mathrm{Na}+\mathrm{K}]<1$ y $[\mathrm{Na}$ $+\mathrm{K}] / \mathrm{Al}<1$, pueden considerarse como magmas de tipo metaluminoso (Figura 6C), lo cual es consistente con la ausencia de corindón normativo (Tabla 2). En contraste, los DP ( $\sim 72-73 \%$ de feldespato alcalino) se ubican en el campo peraluminoso e incluyen corindón en su norma $C I P W$.

Las rocas del CPEP presentan patrones de tierras raras, normalizados a condrita (Boynton, 1984): (a) enriquecidos en tierras raras ligeras o LREE (Figura 7A), con una relación $\mathrm{de}(\mathrm{La} / \mathrm{Yb})_{\mathrm{N}}=6.0-11.4$ que, en general, disminuye con el incremento de $\mathrm{SiO}_{2}$ (Figura 7B); (b) una ausencia de anomalías de $\mathrm{Eu}\left(\left[\mathrm{Eu} / \mathrm{Eu}^{*}\right]_{\mathrm{N}} \sim 0.9-1.0 ; \mathrm{Eu}^{*}=\left(\mathrm{Sm}_{\mathrm{N}} * \mathrm{Gd}_{\mathrm{N}}\right)^{1 / 2}\right)$. Sin embargo, el enclave de monzodiorita V1406 muestra una ligera anomalía negativa de $\mathrm{Eu}$, que podría indicar un magma que estuvo en equilibrio con plagioclasa $\left(\mathrm{D}_{\mathrm{Eu}}=1.1\right.$; Rollinson, 1993); (c) un empobrecimiento relativo en GdHo, que es más marcado en las rocas sieníticas, que podría asociarse a una fraccionación de anfíbol $\left(\mathrm{D}_{\mathrm{Gd}-\mathrm{Ho}}\right.$, magmas máficos a intermedios $=1.4-3.0$; magmas félsicos $=10$ 13; Rollinson, 1993); y (d) un patrón relativamente plano hacia las tierras raras pesadas o HREE. Otra observación importante en los patrones normalizados de los magmas del CPEP (Figura 7A) es la disminución de la concentración de tierras raras totales ( $\Sigma \mathrm{REE}$ ) con el aumento de $\mathrm{SiO}_{2}$, lo que descarta la cristalización fraccionada como proceso
Tabla 2. Composición de elementos mayores $(\% \mathrm{~m} / \mathrm{m})$ y norma $C I P W$ para rocas del CPEP.

\begin{tabular}{|c|c|c|c|c|c|c|c|c|c|}
\hline Muestra & R2101 & CA91701 & V1406 & CA82202 & CA91706 & V1601 & R2202 & CA91702 & CA81808 \\
\hline Tipo & $M S P$ & $M S P$ & $M D$ & $C M D$ & $C M D$ & $C M$ & $C M$ & $C M$ & $C M$ \\
\hline Latitud & 717746 & 721109 & 717121 & 718439 & 718153 & 718081 & 720172 & 720864 & 718621 \\
\hline Longitud & 315582 & 317435 & 320882 & 316196 & 316724 & 320057 & 314673 & 317062 & 317521 \\
\hline $\mathrm{SiO}_{2}$ & 46.18 & 45.71 & 52.82 & 51.79 & 48.62 & 53.99 & 53.93 & 51.97 & 51.35 \\
\hline $\mathrm{TiO}_{2}$ & 1.35 & 1.41 & 1.03 & 0.96 & 1.09 & 0.86 & 0.9 & 0.81 & 1.05 \\
\hline $\mathrm{Al}_{2} \mathrm{O}_{3}$ & 15.26 & 15.12 & 16.51 & 17.32 & 15.71 & 17.63 & 18.11 & 17.94 & 17.28 \\
\hline $\mathrm{Fe}_{2} \mathrm{O}_{3}{ }^{\mathrm{T}}$ & 12.99 & 12.44 & 9.27 & 9.19 & 10.09 & 7.52 & 7.4 & 7.57 & 9.14 \\
\hline $\mathrm{MnO}$ & 0.38 & 0.39 & 0.3 & 0.22 & 0.24 & 0.18 & 0.2 & 0.22 & 0.24 \\
\hline $\mathrm{MgO}$ & 3.29 & 3.4 & 4.16 & 2.6 & 4.6 & 2.25 & 2.11 & 2.3 & 2.71 \\
\hline $\mathrm{CaO}$ & 10.88 & 11.24 & 8.35 & 8.87 & 10.22 & 6.88 & 6.8 & 7.43 & 8.73 \\
\hline $\mathrm{Na}_{2} \mathrm{O}$ & 2.66 & 2.65 & 4.4 & 3.42 & 3.1 & 3.52 & 3.84 & 3.48 & 3.66 \\
\hline $\mathrm{K}_{2} \mathrm{O}$ & 3.72 & 3.35 & 2.04 & 4.37 & 3.95 & 5.29 & 4.99 & 5.12 & 3.88 \\
\hline $\mathrm{P}_{2} \mathrm{O}_{5}$ & 0.71 & 0.66 & 0.5 & 0.54 & 0.88 & 0.46 & 0.39 & 0.46 & 0.59 \\
\hline LOI & 2.1 & 2.11 & 0.53 & 0.32 & 1.31 & 0.23 & 0.4 & 1.34 & 0.42 \\
\hline Total & 99.52 & 98.48 & 99.91 & 99.6 & 99.81 & 98.81 & 99.07 & 98.64 & 99.05 \\
\hline \multicolumn{10}{|c|}{ Norma $C I P W$} \\
\hline$O r$ & 22.752 & 20.713 & 12.198 & 26.15 & 23.846 & 31.841 & 30.003 & 31.227 & 23.373 \\
\hline$A b$ & 10.322 & 10.784 & 37.672 & 22.94 & 14.37 & 28.322 & 29.396 & 23.144 & 25.742 \\
\hline$A n$ & 19.37 & 20.368 & 19.501 & 19.237 & 17.654 & 16.991 & 17.747 & 18.792 & 19.63 \\
\hline $\mathrm{Ne}$ & 7.028 & 6.865 & - & 3.447 & 6.728 & 1.09 & 1.985 & 3.928 & 3.158 \\
\hline$D i$ & 26.176 & 27.583 & 15.435 & 17.735 & 22.821 & 11.903 & 11.318 & 13.101 & 16.77 \\
\hline$H y$ & - & & 2.868 & - & - & - & - & - & - \\
\hline $\mathrm{Ol}$ & 2.606 & 2.322 & 3.628 & 1.541 & 4.339 & 1.952 & 1.814 & 1.996 & 2.147 \\
\hline$M t$ & 7.39 & 6.964 & 5.545 & 5.838 & 6.031 & 5.155 & 5.069 & 5.11 & 5.748 \\
\hline Il & 2.653 & 2.801 & 1.982 & 1.846 & 2.127 & 1.66 & 1.747 & 1.601 & 2.04 \\
\hline$A p$ & 1.703 & 1.599 & 1.172 & 1.267 & 2.083 & 1.084 & 0.92 & 1.1 & 1.392 \\
\hline Mg\# & 44.693 & 46.182 & 60.018 & 49.696 & 60.228 & 52.53 & 51.335 & 52.31 & 50.556 \\
\hline $\mathrm{FeO}^{\mathrm{T}} / \mathrm{MgO}$ & 3.553 & 3.292 & 2.005 & 3.181 & 1.974 & 3.007 & 3.155 & 2.961 & 3.035 \\
\hline$n-\mathrm{Fe}$ & 0.688 & 0.675 & 0.546 & 0.643 & 0.541 & 0.617 & 0.628 & 0.619 & 0.635 \\
\hline MALI & -4.659 & -5.483 & -1.932 & -1.093 & -3.238 & 1.965 & 2.065 & 1.208 & -1.212 \\
\hline ASI & 0.558 & 0.547 & 0.687 & 0.67 & 0.584 & 0.751 & 0.767 & 0.741 & 0.68 \\
\hline$(\mathrm{Na}+\mathrm{K}) / \mathrm{Al}$ & 0.551 & 0.528 & 0.572 & 0.598 & 0.597 & 0.653 & 0.647 & 0.628 & 0.592 \\
\hline
\end{tabular}

\begin{tabular}{|c|c|c|c|c|c|c|c|c|c|}
\hline Muestra & CA42008 & CA12104 & CA42006 & CA81806 & CA42001 & CA51907 & $\begin{array}{c}\text { CA10210 } \\
3\end{array}$ & R2009 & CA 8220 \\
\hline Tipo & $C M$ & $L C S P$ & $L C S P$ & $L C S P$ & LCSP & LCSM & $L C S M$ & $D P$ & $D P$ \\
\hline Latitud & 721226 & 721984 & 721098 & 719176 & 722783 & 719346 & 721145 & 719786 & 715051 \\
\hline Longitud & 318034 & 316705 & 318846 & 317063 & 320867 & 320387 & 315117 & 315760 & 314039 \\
\hline $\mathrm{SiO}_{2}$ & 58.23 & 55.08 & 59.64 & 57.35 & 59.56 & 64.51 & 61.65 & 72 & 70.19 \\
\hline $\mathrm{TiO}_{2}$ & 0.68 & 0.708 & 0.554 & 0.641 & 0.663 & 0.4 & 0.607 & 0.168 & 0.171 \\
\hline $\mathrm{Al}_{2} \mathrm{O}_{3}$ & 18.11 & 20.41 & 18.11 & 18.97 & 17.89 & 16.96 & 17.31 & 14.77 & 15.2 \\
\hline $\mathrm{Fe}_{2} \mathrm{O}_{3} \mathrm{~T}$ & 6.01 & 6.38 & 4 & 5.29 & 5.27 & 3.33 & 4.89 & 1.02 & 1.3 \\
\hline $\mathrm{MnO}$ & 0.154 & 0.19 & 0.103 & 0.157 & 0.151 & 0.1 & 0.157 & 0.016 & 0.016 \\
\hline $\mathrm{MgO}$ & 1.29 & 1.09 & 0.91 & 1.32 & 1.28 & 0.78 & 1.13 & 0.24 & 0.23 \\
\hline $\mathrm{CaO}$ & 5.14 & 5.65 & 4.33 & 5.44 & 5.13 & 3.79 & 5.57 & 1.1 & 1.1 \\
\hline $\mathrm{Na}_{2} \mathrm{O}$ & 4.47 & 5.42 & 4.69 & 4.99 & 4.79 & 4.35 & 4.14 & 4.5 & 4.54 \\
\hline $\mathrm{K}_{2} \mathrm{O}$ & 5.34 & 3.66 & 5.61 & 4.06 & 5.08 & 3.82 & 2.56 & 4.99 & 5.08 \\
\hline $\mathrm{P}_{2} \mathrm{O}_{5}$ & 0.3 & 0.24 & 0.19 & 0.25 & 0.25 & 0.16 & 0.2 & 0.07 & 0.06 \\
\hline LOI & 0.31 & 0.94 & 0.26 & 0.18 & 0.32 & 0.43 & 0.26 & 0.49 & 0.53 \\
\hline Total & 100.04 & 99.77 & 98.4 & 98.65 & 100.38 & 98.63 & 98.47 & 99.36 & 98.42 \\
\hline \multicolumn{10}{|c|}{ Norma $C I P W$} \\
\hline$Q z$ & - & 1.138 & 2.335 & 0.861 & 1.995 & 16.133 & 15.288 & 24.484 & 22.235 \\
\hline Or & 21.96 & 31.74 & 33.85 & 24.436 & 30.08 & 23.024 & 15.448 & 29.838 & 30.689 \\
\hline$A b$ & 40.888 & 38.043 & 40.514 & 43.002 & 40.608 & 37.544 & 35.768 & 38.526 & 39.271 \\
\hline$A n$ & 20.864 & 13.653 & 12.041 & 17.688 & 12.335 & 15.781 & 21.527 & 5.058 & 5.178 \\
\hline $\mathrm{Ne}$ & 3.075 & - & - & - & - & - & - & - & - \\
\hline C & - & - & - & - & - & - & - & 0.136 & 0.383 \\
\hline$D i$ & 4.93 & 8.11 & 6.651 & 6.586 & 9.23 & 1.889 & 4.373 & - & - \\
\hline Hy & - & 0.895 & - & 1.821 & - & 2.043 & 2.688 & 0.639 & 0.709 \\
\hline $\mathrm{Ol}$ & 1.895 & - & - & - & - & - & - & - & - \\
\hline Mt & 4.457 & 4.417 & 3.083 & 3.776 & 3.911 & 2.434 & 3.257 & 0.831 & 1.061 \\
\hline Il & 1.365 & 1.304 & 1.075 & 1.24 & 1.261 & 0.775 & 1.177 & 0.323 & 0.332 \\
\hline$A p$ & 0.565 & 0.7 & 0.449 & 0.588 & 0.579 & 0.378 & 0.473 & 0.164 & 0.141 \\
\hline Mg\# & 39.189 & 46.147 & 48.463 & 48.905 & 49.579 & 47.857 & 45.439 & 51.191 & 43.777 \\
\hline $\mathrm{FeOT} / \mathrm{MgO}$ & 5.266 & 4.194 & 3.956 & 3.606 & 3.706 & 3.84 & 3.892 & 3.821 & 5.092 \\
\hline$n-\mathrm{Fe}$ & 0.734 & 0.675 & 0.655 & 0.651 & 0.644 & 0.66 & 0.682 & 0.63 & 0.696 \\
\hline MALI & 3.483 & 4.697 & 6.095 & 3.677 & 4.749 & 4.467 & 1.154 & 8.489 & 8.71 \\
\hline ASI & 0.893 & 0.819 & 0.845 & 0.855 & 0.798 & 0.943 & 0.889 & 1.003 & 1.02 \\
\hline$(\mathrm{Na}+\mathrm{K}) / \mathrm{Al}$ & 0.631 & 0.725 & 0.761 & 0.664 & 0.748 & 0.666 & 0.554 & 0.867 & 0.853 \\
\hline
\end{tabular}

Para explicación de los acrónimos utilizados consultar la Tabla 1. Mg\# $=100 * \mathrm{Mg}+2 /(\mathrm{Mg}+2+0.9[\mathrm{Fe}+2+\mathrm{Fe}+3])$, atómico; composición de minerales normativos (\%) determinada con la norma CIPW, aplicando el programa SINCLAS (Verma et al., 2002; 2003). Parámetros de clasificación de granitoides (Frost et al., 2001): $\mathrm{n}-\mathrm{Fe}=\mathrm{FeO} /(\mathrm{FeO}+\mathrm{MgO})$; MALI (Modified Alkali-Lime Index) $=\mathrm{Na} 2 \mathrm{O}+\mathrm{K} 2 \mathrm{O}-\mathrm{CaO}$; ASI (Aluminium Saturation Index $)=\mathrm{Al} /(\mathrm{Ca}-1.67 \mathrm{P}+\mathrm{Na}+\mathrm{K})$, molecular 
Tabla 3. Composición de elementos traza (ppm) para rocas del intrusivo CPEP

\begin{tabular}{|c|c|c|c|c|c|c|c|c|c|}
\hline Muestra & R2101 & CA91701 & V1406 & CA82202 & CA91706 & V1601 & R2202 & CA91702 & CA81808 \\
\hline Tipo & $M S P$ & $M S P$ & $M D$ & $C M D$ & $C M D$ & $C M$ & $C M$ & $C M$ & $C M$ \\
\hline $\mathrm{v}$ & 467 & 467 & 220 & 275 & 333 & 207 & 201 & 232 & 270 \\
\hline $\mathrm{Cr}$ & - & - & 80 & - & 50 & - & - & - & - \\
\hline Co & 35 & 34 & 26 & 22 & 33 & 18 & 19 & 21 & 25 \\
\hline $\mathrm{Cu}$ & 210 & 190 & 50 & 160 & 80 & 60 & 50 & 130 & 130 \\
\hline $\mathrm{Zn}$ & 230 & 180 & 160 & 140 & 170 & 120 & 110 & 130 & 130 \\
\hline Ga & 25 & 25 & 26 & 22 & 21 & 20 & 23 & 21 & 23 \\
\hline $\mathrm{Rb}$ & 90 & 78 & 102 & 118 & 104 & 139 & 128 & 132 & 109 \\
\hline $\mathrm{Sr}$ & 1757 & 1677 & 545 & 2192 & 1580 & 1469 & 1417 & 1785 & 1472 \\
\hline $\mathrm{Y}$ & 42.2 & 45.8 & 23.7 & 25.7 & 24.3 & 23.6 & 37.8 & 25.3 & 31.1 \\
\hline $\mathrm{Zr}$ & 193 & 172 & 182 & 125 & 100 & 78 & 114 & 124 & 147 \\
\hline $\mathrm{Nb}$ & 15.3 & 16.8 & 9.6 & 9.2 & 11.3 & 7.7 & 10.4 & 9.6 & 12.8 \\
\hline $\mathrm{Cs}$ & 1.8 & 1.5 & 1.9 & 2.1 & 2.1 & 1.8 & 1.6 & 2.3 & 1.4 \\
\hline $\mathrm{Ba}$ & 1250 & 1089 & 397 & 2302 & 964 & 1277 & 963 & 1052 & 853 \\
\hline $\mathrm{La}$ & 57.8 & 61.4 & 36 & 29.9 & 33.5 & 24.4 & 28.4 & 30.9 & 42.4 \\
\hline $\mathrm{Ce}$ & 120 & 128 & 59.2 & 61.9 & 65 & 52.4 & 61.6 & 63.7 & 82.3 \\
\hline $\mathrm{Pr}$ & 16.6 & 17.8 & 6.93 & 8.45 & 8.97 & 7.29 & 8.65 & 8.5 & 10.4 \\
\hline $\mathrm{Nd}$ & 65.5 & 70.3 & 24.6 & 34.6 & 35.6 & 32.6 & 34.9 & 36.6 & 44.2 \\
\hline $\mathrm{Sm}$ & 15.3 & 16.4 & 5.32 & 8.21 & 8.55 & 7.25 & 8.31 & 7.58 & 9.34 \\
\hline Eu & 4.27 & 4.61 & 1.27 & 2.61 & 2.54 & 2.22 & 2.49 & 2.27 & 2.69 \\
\hline $\mathrm{Gd}$ & 12.2 & 13.1 & 4.69 & 6.73 & 7.08 & 6.15 & 6.87 & 6.37 & 7.36 \\
\hline $\mathrm{Tb}$ & 1.55 & 1.66 & 0.66 & 0.88 & 0.85 & 0.82 & 0.93 & 0.84 & 1.01 \\
\hline Dy & 7.68 & 8.4 & 3.76 & 4.66 & 4.48 & 4.33 & 5.03 & 4.51 & 5.39 \\
\hline Ho & 1.45 & 1.56 & 0.77 & 0.89 & 0.85 & 0.83 & 0.98 & 0.88 & 1.05 \\
\hline $\mathrm{Er}$ & 4.08 & 4.4 & 2.36 & 2.53 & 2.45 & 2.39 & 2.81 & 2.55 & 3.11 \\
\hline $\mathrm{Tm}$ & 0.574 & 0.64 & 0.359 & 0.366 & 0.347 & 0.338 & 0.401 & 0.37 & 0.469 \\
\hline $\mathrm{Yb}$ & 3.5 & 3.93 & 2.33 & 2.29 & 2.11 & 1.99 & 2.46 & 2.3 & 2.99 \\
\hline $\mathrm{Lu}$ & 0.527 & 0.562 & 0.355 & 0.33 & 0.31 & 0.289 & 0.355 & 0.323 & 0.428 \\
\hline $\mathrm{Hf}$ & 5.3 & 5 & 4.2 & 3.2 & 2.6 & 2.3 & 3.1 & 3.2 & 4 \\
\hline $\mathrm{Ta}$ & 1.23 & 1.41 & 0.68 & 0.67 & 5.71 & 0.71 & 0.9 & 0.72 & 0.8 \\
\hline $\mathrm{Pb}$ & 13 & 8 & 10 & 9 & 11 & 12 & 13 & 15 & 9 \\
\hline Th & 6.36 & 6.51 & 6.46 & 4.86 & 4.66 & 3.38 & 3.85 & 4.81 & 5.69 \\
\hline $\mathrm{U}$ & 1.9 & 1.97 & 2.52 & 1.92 & 2.14 & 1.23 & 1.6 & 1.9 & 2.37 \\
\hline Muestra & CA42008 & CA12104 & CA42006 & CA81806 & CA42001 & CA51907 & CA102103 & R2009 & CA82201 \\
\hline Tipo & $C M$ & ${ }_{L C S P}$ & $L C S P$ & $L C S P$ & LCSP & $L C S M$ & $L C S M$ & $D P$ & $D P$ \\
\hline $\mathrm{v}$ & 164 & 143 & 105 & 134 & 135 & 64 & 72 & 41 & 39 \\
\hline $\mathrm{Cr}$ & 16 & 16 & 7 & 9 & 12 & 6 & 10 & 1 & 3 \\
\hline Co & - & - & - & - & - & 570 & - & - & - \\
\hline $\mathrm{Cu}$ & 60 & 40 & - & 10 & 25 & 10 & - & - & - \\
\hline $\mathrm{Zn}$ & 140 & 110 & 70 & 80 & 80 & 130 & 60 & - & 180 \\
\hline Ga & 28 & 23 & 23 & 24 & 25 & 22 & 21 & 18 & 21 \\
\hline $\mathrm{Rb}$ & 99 & 175 & 174 & 116 & 182 & 156 & 92 & 118 & 141 \\
\hline $\mathrm{Sr}$ & 1527 & 1132 & 1058 & 1583 & 1074 & 779 & 795 & 444 & 466 \\
\hline $\mathrm{Y}$ & 26.7 & 26.5 & 24.3 & 26 & 28.4 & 16.7 & 32.1 & 10.3 & 11.9 \\
\hline $\mathrm{Zr}$ & 123 & 194 & 149 & 210 & 180 & 174 & 205 & 132 & 147 \\
\hline $\mathrm{Nb}$ & 15.1 & 11.2 & 11.2 & 12.3 & 11.8 & 8.9 & 9.8 & 6 & 6.5 \\
\hline $\mathrm{Cs}$ & 1.8 & 2.4 & 2 & 1.7 & 2.3 & 1.9 & 1 & 0.7 & 0.8 \\
\hline $\mathrm{Ba}$ & 594 & 733 & 720 & 1503 & 709 & 734 & 572 & 675 & 692 \\
\hline $\mathrm{La}$ & 39.1 & 35.2 & 29.7 & 34.3 & 33.4 & 25.2 & 30.1 & 22.1 & 18.8 \\
\hline $\mathrm{Ce}$ & 77.6 & 65.5 & 59.88 & 68.2 & 64.8 & 42.9 & 59.1 & 32.3 & 27.6 \\
\hline $\operatorname{Pr}$ & 10.1 & 8.19 & 7.57 & 8.71 & 8.38 & 4.91 & 7.35 & 3.28 & 2.72 \\
\hline $\mathrm{Nd}$ & 41.1 & 30.4 & 30.4 & 32.7 & 29.4 & 19.1 & 27.7 & 9.92 & 8.99 \\
\hline $\mathrm{Sm}$ & 8.18 & 6.89 & 6.06 & 7.37 & 6.43 & 3.74 & 6.33 & 1.81 & 1.82 \\
\hline Eu & 2.34 & 2.02 & 1.66 & 2.24 & 2 & 1.18 & 1.86 & 0.538 & 0.557 \\
\hline $\mathrm{Gd}$ & 6.49 & 5.81 & 4.98 & 6.07 & 5.41 & 3.03 & 5.72 & 1.36 & 1.66 \\
\hline $\mathrm{Tb}$ & 0.87 & 0.81 & 0.69 & 0.82 & 0.76 & 0.45 & 0.85 & 0.25 & 0.25 \\
\hline Dy & 4.67 & 4.4 & 3.85 & 4.34 & 4.16 & 2.57 & 5.04 & 1.63 & 1.55 \\
\hline Но & 0.91 & 0.9 & 0.79 & 0.88 & 0.85 & 0.55 & 1.06 & 0.36 & 0.35 \\
\hline $\mathrm{Er}$ & 2.68 & 2.72 & 2.44 & 2.66 & 2.69 & 1.69 & 3.37 & 1.15 & 1.18 \\
\hline $\mathrm{Tm}$ & 0.383 & 0.437 & 0.389 & 0.404 & 0.413 & 0.269 & 0.518 & 0.201 & 0.207 \\
\hline $\mathrm{Yb}$ & 2.32 & 2.89 & 2.54 & 2.64 & 2.64 & 1.87 & 3.37 & 1.5 & 1.55 \\
\hline Lu & 0.323 & 0.422 & 0.391 & 0.387 & 0.393 & 0.319 & 0.508 & 0.27 & 0.269 \\
\hline $\mathrm{Hf}$ & 3.2 & 4.8 & 3.9 & 4.6 & 4.3 & 4.4 & 5.2 & 4.6 & 4 \\
\hline $\mathrm{Ta}$ & 1.06 & 0.81 & 0.76 & 0.92 & 0.82 & 0.57 & 0.87 & 0.47 & 0.47 \\
\hline $\mathrm{Pb}$ & 16 & 11 & 15 & 12 & 10 & 18 & - & 11 & 12 \\
\hline Th & 7.98 & 9.1 & 8.34 & 7.75 & 9.52 & 8.7 & 7.21 & 14.4 & 13.1 \\
\hline $\mathrm{U}$ & 2.81 & 3.62 & 2.92 & 2.81 & 3.31 & 2.49 & 2.41 & 2.8 & 2.61 \\
\hline
\end{tabular}

Para explicación de los acrónimos utilizados consultar la Tabla 1.

dominante de evolución magmática.

La descripción de las características geoquímicas de las rocas analizadas se complementa con los patrones multielementos normalizados al manto primordial (Palme y O’Neill, 2003). Estos patrones se caracterizan por mostrar
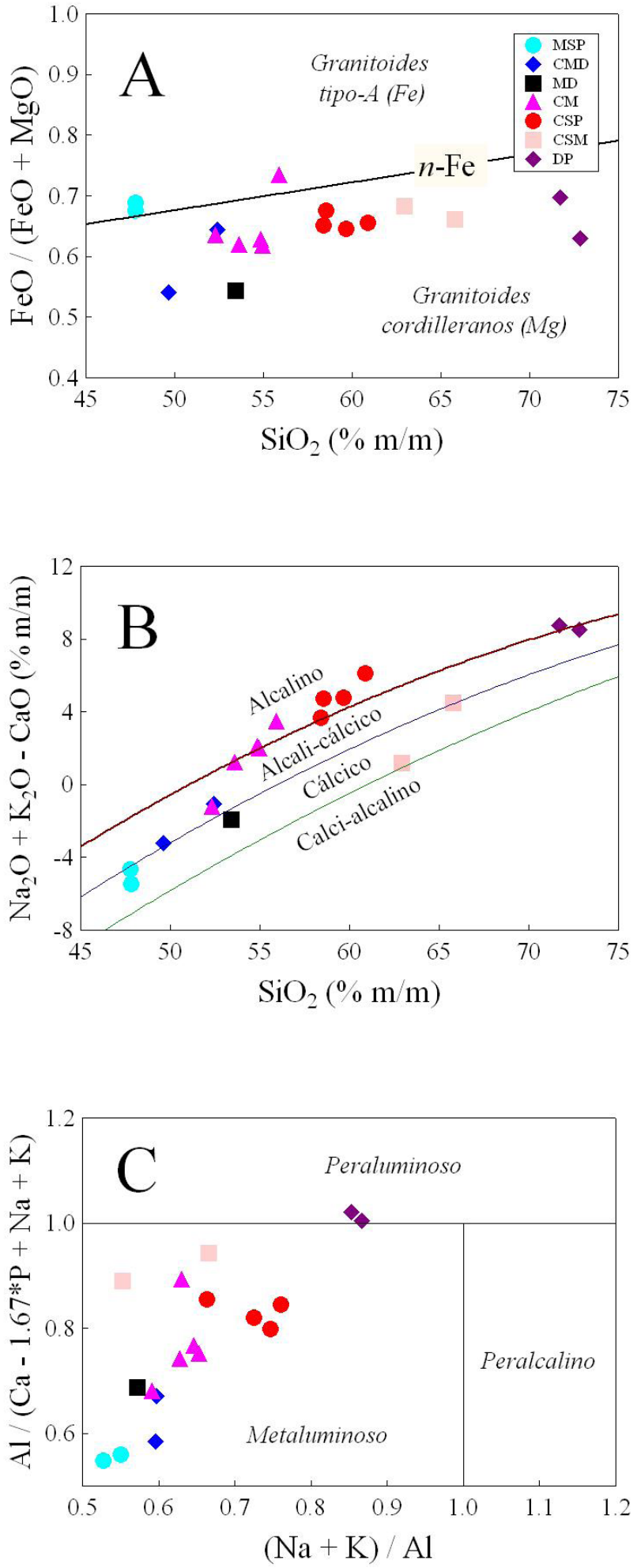

Figura 6. Diagramas de clasificación de Frost et al. (2001) para las rocas del CPEP: (A) Diagrama $n$ - $\mathrm{Fe}[\mathrm{FeO} /(\mathrm{FeO}+\mathrm{MgO})](\% \mathrm{~m} / \mathrm{m})-\mathrm{SiO}_{2}(\% \mathrm{~m} / \mathrm{m})$; (B) Diagrama $\left(\mathrm{Na}_{2} \mathrm{O}+\mathrm{K}_{2} \mathrm{O}-\mathrm{CaO}\right)(\% \mathrm{~m} / \mathrm{m})-\mathrm{SiO}_{2}(\% \mathrm{~m} / \mathrm{m})$; (C) Diagrama $[\mathrm{Al} /(\mathrm{Ca}-1.67 \mathrm{P}+\mathrm{Na}+\mathrm{K})](\mathrm{mol})-[(\mathrm{Na}+\mathrm{K}) / \mathrm{Al}](\mathrm{mol})$. Para símbolos y muestras consultar la Tabla 1 y las Figuras 2 y 3 . Para explicación de los acrónimos utilizados consultar la Figura 2. 

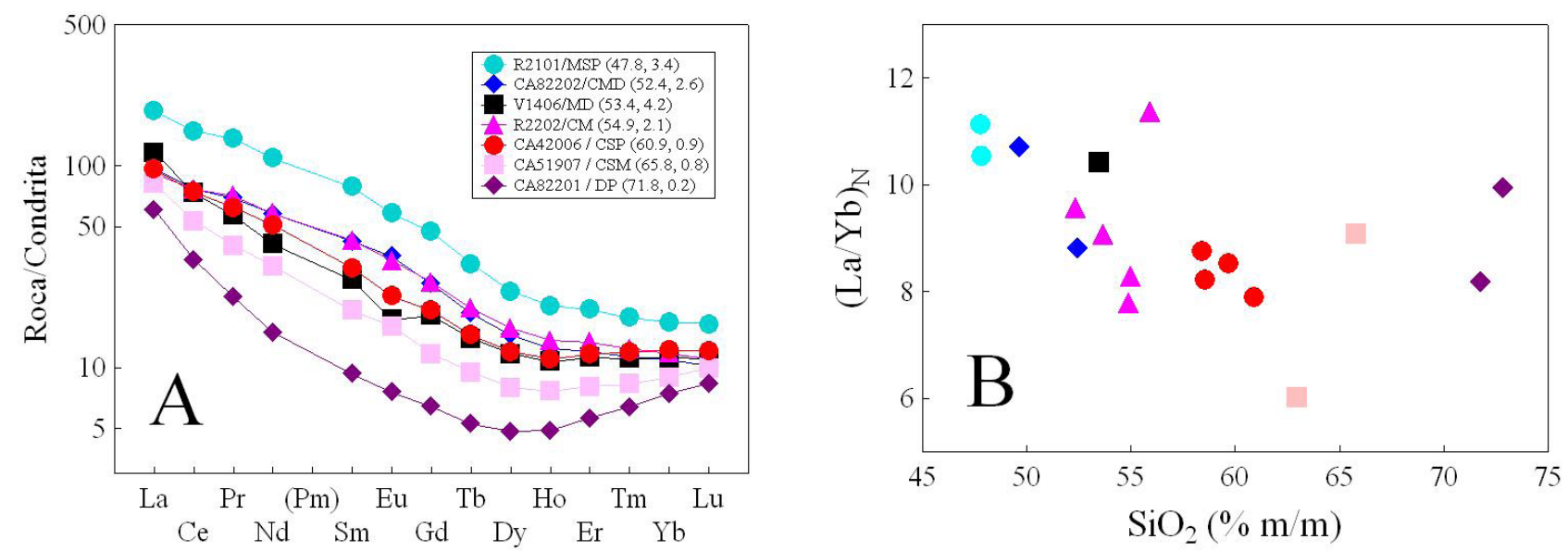

Figura 7. (A) Diagramas de tierras raras, normalizados a condrita para las rocas del CPEP. Datos de normalización en ppm (Boynton, 1984): La $=0.3100$, $\mathrm{Ce}=0.8080, \mathrm{Pr}=0.1120, \mathrm{Nd}=0.6000, \mathrm{Sm}=0.1950, \mathrm{Eu}=0.0735, \mathrm{Gd}=0.2590, \mathrm{~Tb}=0.0474, \mathrm{Dy}=0.3220, \mathrm{Ho}=0.0718, \mathrm{Er}=0.2100, \mathrm{Tm}=0.0324, \mathrm{Yb}$ $=0.2090, \mathrm{Lu}=0.0322$. Los valores entre paréntesis indican la concentración de $\mathrm{SiO}_{2}$ y $\mathrm{MgO}(\% \mathrm{~m} / \mathrm{m})$. (B) Diagrama de $(\mathrm{La} / \mathrm{Yb})_{\mathrm{N}}-\mathrm{SiO}{ }_{2}(\% \mathrm{~m} / \mathrm{m}) \mathrm{para}$ las diversas litologías que constituyen el CPEP. Para explicación de los acrónimos utilizados consultar la Figura 2.

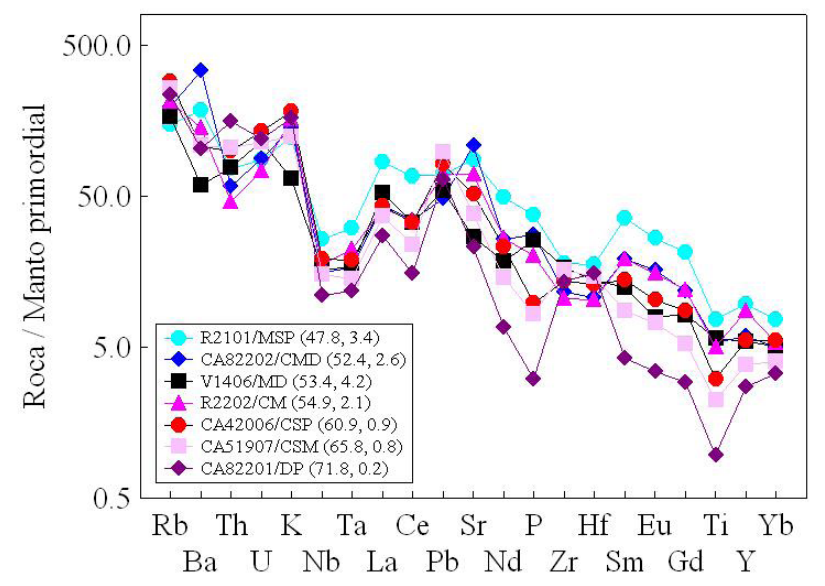

Figura 8. Diagramas multi-elementos, normalizados a manto primordial para las rocas del CPEP. Datos de normalización en ppm (Palme y O’Neill, 2003): $\mathrm{Rb}=0.605, \mathrm{Ba}=6.75, \mathrm{Th}=0.0834, \mathrm{U}=0.0218, \mathrm{~K}=260, \mathrm{Nb}=$ $0.588, \mathrm{Ta}=0.04, \mathrm{La}=0.686, \mathrm{Ce}=1.786, \mathrm{~Pb}=0.185, \mathrm{Sr}=20.3, \mathrm{Nd}=$ $1.327, \mathrm{P}=86, \mathrm{Zr}=10.81, \mathrm{Hf}=0.3, \mathrm{Sm}=0.431, \mathrm{Eu}=0.162, \mathrm{Gd}=0.571$, $\mathrm{Ti}=1280, \mathrm{Y}=4.37, \mathrm{Yb}=0.462$. Los valores entre paréntesis indican la concentración de $\mathrm{SiO}_{2}$ y $\mathrm{MgO}$ (\% m/m aj). Para explicación de los acrónimos utilizados consultar la Figura 2

un enriquecimiento con respecto al manto primordial (Figura 8), especialmente de elementos altamente incompatibles (por ejemplo, $\mathrm{Rb}$ y $\mathrm{Sr}$ ), con un descenso general en concentración conforme se incrementa la compatibilidad. Los patrones de todas las unidades litológicas muestran anomalías negativas de elementos incompatibles de campo electrostático fuerte, tal como $\mathrm{Nb}$ - $\mathrm{Ta}$ y $\mathrm{Ti}$, siendo más marcadas en las rocas relativamente más félsicas (CSP, CSM y DP) y que también incluyen una anomalía negativa muy marcada de fósforo. Al igual que lo observado en los diagramas de tierras raras, se registra una disminución en concentración de las litologías máficas a félsicas.

\section{Modelo de génesis magmática}

Aunque los granitoides representan el tipo de roca más abundante en la corteza continental (Clarke, 1992; Rudnick y Gao, 2003), su petrogénesis es un tema complejo (e.g., Chappell y White, 1974; Reverdatto y Kalinin, 1980; Castro, 1987; Borodin, 1988; Maniar y Piccoli, 1989; Barbarin, 1990, 1999; Frost et al., 2001) e involucra diversos factores que incluyen el ambiente tectónico, el tipo de fuente inicial del magmatismo (manto y/o corteza), las condiciones de fusión parcial y los procesos de evolución magmática, tales como cristalización fraccionada, cristalización fraccionada con asimilación de material cortical y mezcla de magmas.

De acuerdo a la clasificación de Frost et al. (2001), las características geoquímicas del CPEP corresponden a granitoides de tipo cordillerano (tipo-I según la clasificación clásica de Chappell y White, 1974): (a) son dominantemente magnésicos (Figura 6A; Mg\#>39, Tabla 1), (b) presentan características cálcicas a alcalinas (Figura 6B), que son típicas para plutones ubicados en las partes internas de cinturones batolíticos (Bateman y Dodge, 1970); y (c) presentan un carácter metaluminoso (con excepción de los DP; Figura 6C), congruente con la presencia de anfíboles y clinopiroxenos, así como con la ausencia de muscovita o fases ferromagnesianas sódicas (Tabla 1). Esto es consistente con la ausencia de coridón normativo, característico de rocas hiperaluminosas, como se observa para los DP (Tabla 2).

El origen de los granitoides cordilleranos ha sido asociado a ambientes tectónicos de arco (Chappell y White, 1974; Barbarin, 1999). Diagramas de discriminación 
indican, con la excepción de MD y MSP, una asociación de las rocas intrusivas del CPEP a granitoides tipo I (Figura 9A), que son generados en un ambiente de arco continental normal (Figura 9B y D). Una relación $\mathrm{Ta} / \mathrm{Yb}>0.1$ (Figura 9C) es típica para magmas de arco continental (Pearce et al., 1984). Por otra parte, Brown et al. (1984) demostraron que el incremento de la relación $\mathrm{Rb} / \mathrm{Zr}$ con el aumento de $\mathrm{Nb}$ e Y corresponde al grado de madurez de un arco. En el caso del CPEP, una relación $\mathrm{Rb} / \mathrm{Zr}<1.8$ (Figura 9D) es indicativo de un arco en condiciones normales de madurez.

Sin embargo, en contraste con lo observado en granitoides tipo I típicos, las rocas intrusivas del CPEP presentan: (a) una alta concentración de $\mathrm{Ba}$ (= 594-2302 ppm) y $\operatorname{Sr}(=444-2192$ ppm); (b) una baja concentración de Y (=10-46 ppm) y Nb (=6-17 ppm); y (c) un alto valor para las relaciones $\mathrm{Sr} / \mathrm{Y}(=25-85)$ y $\mathrm{La} / \mathrm{Yb}(=8.9-16.5)$.
Estas características son comparables a las reportadas para granitoides post-orogénicos de alto $\mathrm{Ba}-\mathrm{Sr}$, tal como se observa en las Figuras 9B (Pearce, 1996) y 10 (Tarney y Jones, 1994; Fowler y Henney, 1996; Fowler et al., 2001). La literatura señala que estos granitoides se originan por fusión parcial de un manto litosférico enriquecido, en condiciones post-orogénicas, seguida de asimilación cortical (Tarney y Jones, 1994; Qian et al., 2003; Fowler et al., 2008).

Este modelo es consistente con el marco tectónico de la región en el Cretácico Superior: una subducción subhorizontal de la placa Farallón bajo Norteamérica, que resultó en la migración de la actividad magmática hacia el E del continente (Clark et al., 1982; Humpreys et al., 2003). El área en donde se ubica el CPEP se encontraba alejada de la zona de trinchera y la secuencia sedimentaria había
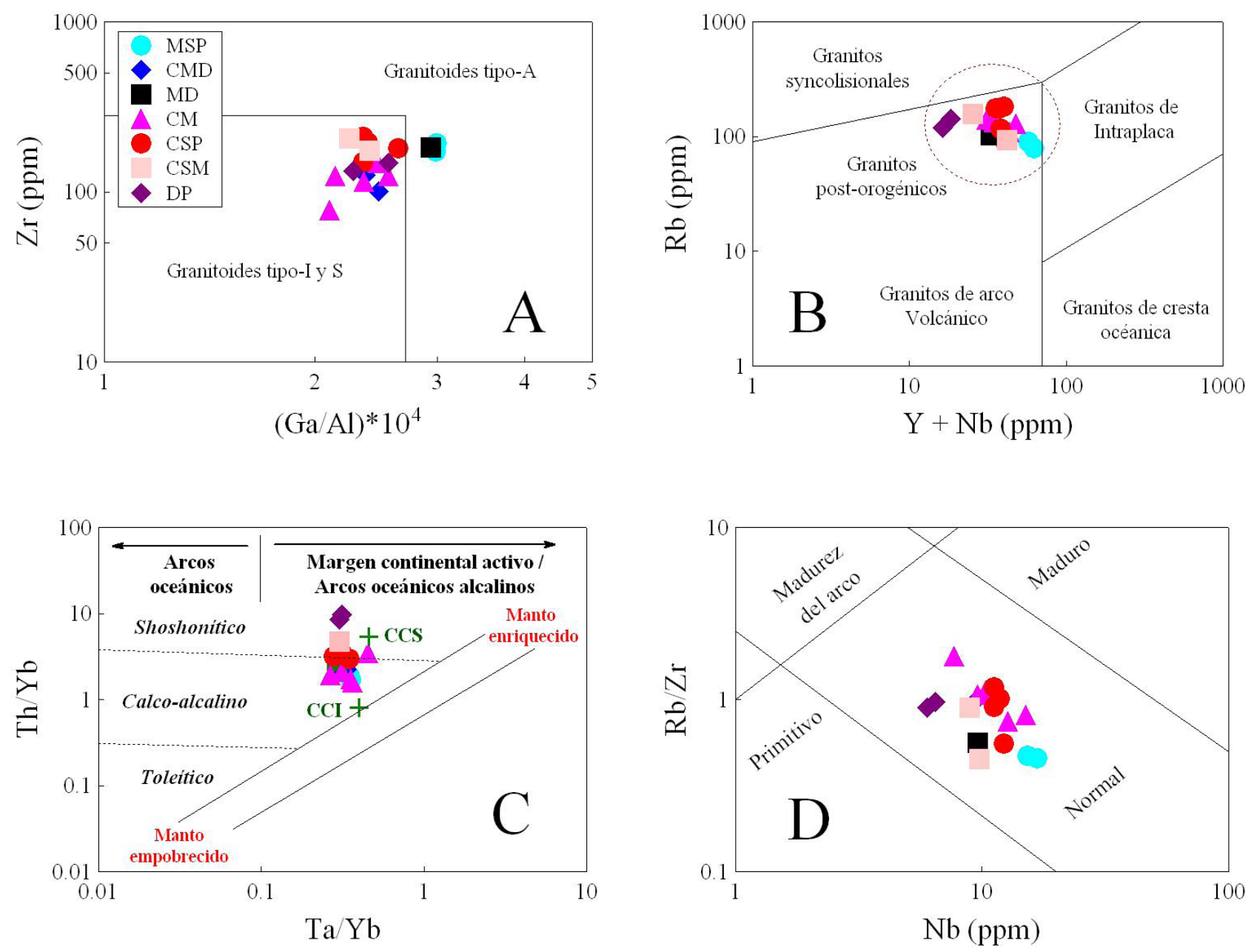

Figura 9. Diagramas bivariados que involucran elementos traza para las rocas del CPEP: (A) Diagrama de discriminación $\mathrm{Zr}-(\mathrm{Ga} / \mathrm{Al}) * 10^{4}$ para granitoides (Whalen et al., 1987). Para explicación de los acrónimos utilizados consultar la Figura 2; (B) Diagrama de discriminación Rb - (Y + Nb) para granitos (Pearce et al., 1984). El diagrama también incluye el campo de granitoides post-orogénicos, propuesto por Pearce (1996); (C) Diagrama de relaciones de elementos inmóviles $\mathrm{Th} / \mathrm{Yb}-\mathrm{Ta} / \mathrm{Yb}$ (Pearce, 1983). CCI = corteza continental inferior y CCS = corteza continental superior (Rudnick y Gao, 2003). Las líneas discontinuas inclinadas reflejan el área que ocupan los magmas en equilibrio con el manto; las líneas discontinuas horizontales indican las fronteras entre magmas toleíticos, calco-alcalinos y shoshoníticos; (D) Diagrama Rb-Zr - Nb para determinación de madurez del arco (Brown et al., 1984) 


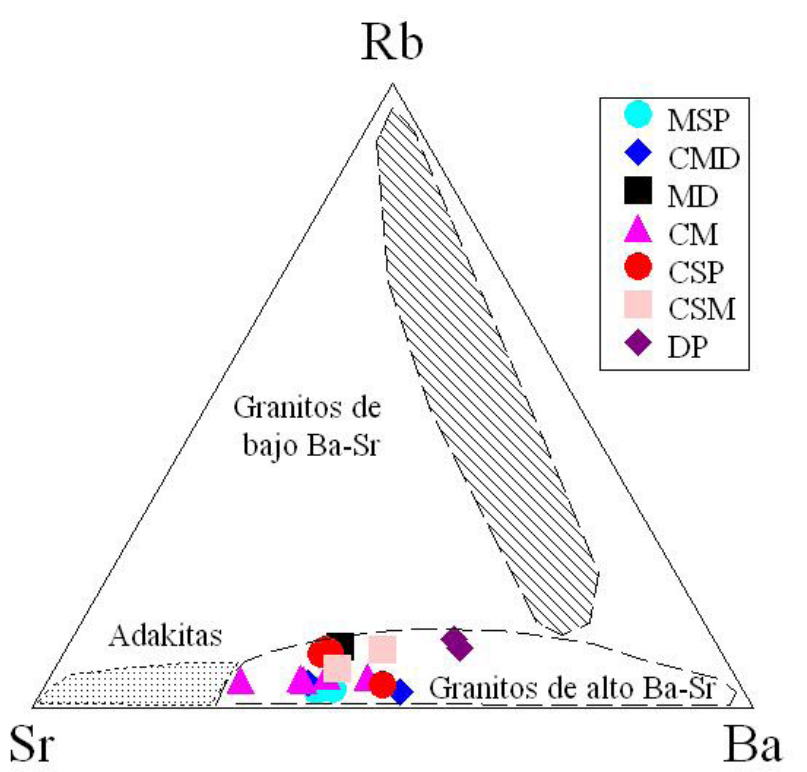

Figura 10. Diagrama ternario Sr-Ba-Rb propuesto por Tarney y Jones (1994) para rocas del CPEP. Los campos de granitoides de alto y bajo Ba-Sr se basan en la información reportada por Fowler y Henney (1996) y Fowler et al. (2001). Para explicación de los acrónimos utilizados consultar la Figura 2.

sido deformada por el evento laramídico e intrusionada por los cuerpos magmáticos. Estos muestran relaciones de corte (post-tectónico) con respecto a los ejes anticlinales regionales en la zona (por ejemplo, el intrusivo Rocamontes; Chávez-Cabello et al., 2007; Delgado-García y MoralesAcosta, 2010). De esta forma, la región podría considerarse en condiciones post-orogénicas.

Las rocas del CPEP con $\mathrm{SiO}_{2}<53 \%$ (MSP, MD, CMD y $\mathrm{CM}$ ) presentan $\mathrm{Mg} \#=44-60$ (Tabla 2), por lo que podrían considerarse como líquidos derivados de la fusión parcial del manto y evolucionados, en principio, por cristalización fraccionada de minerales ferromagnesianos (Rollinson, 1993). Cabe destacar que el alto contenido de $\mathrm{K}_{2} \mathrm{O}=3.5$ $4.0 \%$ (Tabla 1), así como las altas concentraciones en $\mathrm{Sr}$ y Ba (Tabla 2) para estas rocas, podría ser un indicativo de la derivación desde un manto metasomatizado. Durante el proceso de emplazamiento, los líquidos máficos derivados del manto habrían experimentado además un cierto grado de asimilación de material cortical. La CSP, que es la litología más importante a nivel afloramiento, se caracteriza por $\mathrm{SiO}_{2}$ $=55-60 \%$ y Mg\# $=46-50$, acompañados de altos valores de $\mathrm{K}_{2} \mathrm{O}=3.7-5.6 \%$. Esta unidad litológica contiene enclaves microgranitoides de MD y además es cortada por diques de MSP (Figura 2).

Por otra parte, una variación de $\mathrm{Th} / \mathrm{Yb}=1.6-9.6 \mathrm{de}$ rocas máficas a félsicas del CPEP, asociada a una más restringida en $\mathrm{Ta} / \mathrm{Yb}=0.3-0.5$ (Figura $9 \mathrm{C}$ ) es probablemente indicativo de una evolución magmática por cristalización fraccionada acoplada a la asimilación de material cortical. La importancia del proceso de asimilación se refuerza ya que en los diagramas normalizados de tierras raras (Figura 7A) y multi-elementos (Figura 8), no se observa un incremento en las concentraciones de elementos incompatibles con el aumento del $\mathrm{SiO}_{2}$, un fenómeno esperado en sistemas magmáticos que evolucionan sólo por cristalización fraccionada (Rollinson, 1993). De esta forma, los DP que presentan la concentración más alta de $\mathrm{SiO}_{2}(=70-72 \%)$ en el CPEP, tienen las concentraciones más bajas de REE (Figura 7A).

Adicionalmente, el contacto entre las unidades ígneas cartografíadas sugiere que CM, CSP, CSM y los DP, muestran relaciones de corte abruptas que permiten distinguir que su emplazamiento ocurrió en diferentes tiempos y en donde las unidades previamente emplazadas ya se habían enfriado. Sin embargo, la CSP y la CSM muestran contacto transicional, siendo la última una variedad textural de grano más fino de la primera, debido a su enfriamiento más rápido por la relación de contacto directo con las rocas que encajonan al complejo intrusivo.

\section{Conclusiones}

El CPEP, que forma parte del Cinturón de Intrusivos de Concepción del Oro (noreste de México), está constituido de granitoides que cubren un espectro composicional amplio. Estas litologías poseen características petrográficas y geoquímicas de granitoides de alto contenido en Ba y Sr. Durante el Cretácico Superior al Terciario, periodo en el que posiblemente se emplazaron las unidades que componen a este intrusivo, la placa Farallón subducía bajo la costa occidental del norte de México. El origen de los granitoides en esta localidad puede explicarse a partir de un modelo complejo que involucra la fusión parcial del manto litosférico enriquecido, en condiciones post-orogénicas. Durante su ascenso y emplazamiento, los magmas experimentaron un proceso de cristalización fraccionada acoplada con asimilación de material cortical. Para corroborar el modelo conceptual sería necesario contar con información de composición química de minerales, relaciones isotópicas $\mathrm{Sr}-\mathrm{Nd}-\mathrm{Pb}$ y la aplicación de una metodología geocronológica adecuada a las diferentes litologías que conforman el CPEP.

\section{Agradecimientos}

Este trabajo ha sido realizado con fondos CONACyT dentro del proyecto "Magmatismo, deformación y metalogenia Laramide: análisis de la subducción y el papel de la litósfera en el norte de México" (Clave V49528-F). Los autores agradecen los comentarios de Luis A. Delgado Argote y un revisor anónimo, que han permitido mejorar el manuscrito original. 


\section{Referencias}

Alaniz-Álvarez, S.A., Nieto-Samaniego, A.F., Orozco-Esquivel, M.T., Vasallo, L.F., Xu, S., 2002, El sistema de fallas Taxco-San Miguel de Allende: Implicaciones en la deformación post-eocénica del centro de México: Boletín de la Sociedad Geológica Mexicana, 55, 12-29.

Anderson, T.H., Silver, L.T., 1974, Late Cretaceous plutonism in Sonora, Mexico and its relationship to circum-Pacific magmatism: Geological Society of America Abstracts with Programs, 6, 484.

Aranda-Gomez, J.J., Luhr, J.F., Housh, T.B., Valdez-Moreno, G., ChávezCabello, G., 2005, El volcanismo tipo intraplaca del Cenozoico tardío en el centro y norte de México: una revisión: Boletín de la Sociedad Geológica Mexicana, 57, 187-225.

Barbarin, B., 1990, Granitoids: Main petrogenetic classifications in relation to origin and tectonic setting: Geological Journal, 25, 227-238.

Barbarin, B., 1999, A review of the relationships between granitoid types, their origins and their geodynamic environments: Lithos, 46, 605-626.

Bateman, P.C., Dodge, F.C.W., 1970, Variations of major chemical constituents across the central Sierra Nevada batholith: Geological Society of America Bulletin, 81, 409-420.

Borodin, L.S., 1988, Petrochemical trends and classification of the gabbrogranitoid series: International Geology Review, 30, 1189-1198.

Boynton, W.V., 1984, Cosmochemistry of the rare earth elements: Meteorite studies, en Henderson, P. (ed.), Rare Earth Element Geochemistry: Amsterdam, Elsevier, 63-114.

Brown, G.C., Thorpe, R.S., Webb, P.C., 1984, The geochemical characteristics of granitoids in contrasting arcs and comments on magma sources: Journal of the Geological Society, 141, 413-426.

Castro, A., 1987, On granitoid emplacement and related structures, a review: Geologische Rundschau, 76, 101-124.

Castro-Reino, S.F., 2004, Intrusion-related mineralization in the central sector of the Sierra Madre Oriental, Mexico: Tucson, Arizona, E.U.A, University of Arizona, tesis doctoral, $500 \mathrm{p}$.

Chase, T., 1909, Concepción del Oro district, state of Zacatecas, México: Mining World, 31, 1068-1070.

Chappell, B.W., White, A.J.R., 1974, Two contrasting granite types: Pacific Geology, 8, 173-174.

Chávez-Cabello, G., Lozano-Serna, J., Medina-Ferrusquia, H., ValenciaMoreno, M., Velasco-Tapia, F., Montalvo-Arrieta, J.C., Yutsis, V., Navarro-De León, I., 2007, Cartografía e historia de emplazamiento del complejo intrusivo El Peñuelo, en los límites de los estados de Zacatecas, San Luis Potosí, Coahuila y Nuevo León: Geos, 27, 57.

Clark, K.F., Foster, C.T., Damon, P.E., 1982, Cenozoic mineral deposits and subduction-related magmatic arcs in Mexico: Geological Society of America Bulletin, 93, 533-544.

Clarke, D.B., 1992, Granitoid rocks. Topics in the Earth Science 7: Londres, Chapman \& Hall, $283 \mathrm{p}$.

Coney, P.J., Reynolds, S.J., 1977, Cordilleran Benioff zones: Nature, 270, 403-406.

Consejo de Recursos Minerales (COREMI), 1997, Texto guía de la carta magnética de Concepción del Oro G14-10: México, D.F., Secretaría de Minas e Industria Paraestatal, $23 \mathrm{p}$.

Damon, P.E., Shafiqullah, M., Roldán-Quintana, J., Cochemé, J.J., 1983, El batolito Laramide (90-40 Ma) de Sonora (resumen), en Memorias de la XV Convención Nacional de la AIMMGM: Guadalajara, Jalisco, Asociación de Ingenieros de Minas, Metalurgistas y Geólogos de México, 63-95.

De Cserna, Z., 1976, Geology of the Fresnillo area, Zacatecas, Mexico: Geological Society of America Bulletin, 87, 1191-1199.

Delgado-García, S., Morales-Acosta, G., 2010, Cartografía y análisis estructural del intrusivo Rocamontes, Coahuila y Zacatecas, México: Linares, Nuevo León, México, Universidad Autónoma de Nuevo León, tesis de licenciatura, $131 \mathrm{p}$.

Duque-Botero, F., Maurrasse, F.J.-M.R., 2008, Role of cyanobacteria in $\mathrm{C}_{\mathrm{org}}$-rich deposits: an example from the Indidura Formation (Cenomanian-Turonian), northeastern Mexico: Cretaceous Research, $29,957-964$.
Eguiluz de Antuñano, S., Aranda-García, M., Marrett, R., 2000, Tectónica de la Sierra Madre Oriental, México: Boletín de la Sociedad Geológica Mexicana, 53, 1-26.

Elías-Herrera, M., Rubinovich-Kogan, R., Lozano-Santa Cruz, R., Sánchez-Zavala, J.L., 1991, Nepheline-rich foidolites and rare-earth mineralization in the El Picacho Tertiary intrusive complex, Sierra de Tamaulipas, northeastern Mexico: The Canadian Mineralogist, 29, 319-336

Fowler, M.B., Henney, P.J., 1996, Mixed Caledonian appinite magmas: implications for lamprophyre fractionation and high $\mathrm{Ba}-\mathrm{Sr}$ granite genesis: Contributions to Mineralogy and Petrology, 126, 199-215.

Fowler, M.B., Henney, P.J., Darbyshire, D.P.F., Greenwood, P.B., 2001, Petrogenesis of high Ba-Sr granites: the Rogart pluton, Sutherland: Journal of the Geological Society, 158, 521-534.

Fowler, M.B., Kocks, H., Darbyshire, D.P.F., Greenwood, P.B., 2008, Petrogenesis of high Ba-Sr plutons from the Northern Terrene of the British Caledonian Province: Lithos, 105, 129-148.

Frost, B.R., Barnes C.G., Collins W.J., Arculus R.J., Ellis D.J., Frost C.D., 2001. A geochemical classification for granitic rocks: Journal of Petrology, 42, 2033-2048.

González León, C.M., McIntosh, W.C., Lozano-Santacruz, R., ValenciaMoreno, M., Amaya-Martínez, R., Rodríguez-Castañeda, J.L., 2000, Cretaceous and Tertiary sedimentary, magmatic, and tectonic evolution of north-central Sonora (Arizpe and Bacanuchi Quadrangles), northwest Mexico: Geological Society of America Bulletin, 112, 600-610.

Hoffer, J.M., 1970, Petrology and mineralogy of the Campus Andesite Pluton, El Paso, Texas: Geological Society of America Bulletin, 81, 2129-2136.

Humpreys, E., Hessler, E., Dueker, K., Farmer, G.L., Erslev, E., Atwater, T., 2003, How Laramide-age hydration of North American lithosphere by the Farallon slab controlled subsequent activity in the western United States: International Geology Review, 45, 575-595.

Jones, T.S., 1938, Geology of the Sierra de la Pena and paleontology of the Indidura Formation, Coahuila, Mexico: Geological Society of America Bulletin, 49, 69-149.

Kelly, W.A., 1936, Evolution of the Coahuila Peninsula, Mexico; Part 2, Geology of the mountain bordering the valleys of Acatita and Las Delicias: Geological Society of America Bulletin, 47, 1009-1038.

Krieger, P., 1940, Bornite-Klaprotholite relations at Concepción del Oro, Mexico: Economic Geology, 35, 687-697.

Le Maitre, R.W., Streckeisen, A., Zanettin, B., Le Bas, M.J., Bonin, B., Bateman, P., Bellieni, G., Dudek, A., Efremova, S., Keller, J., Lameyre, J., Sabine, P.A., Schmid, R., Sorensen, H., Woolley, A.R., 2002, Igneous Rocks: A classification and glossary of terms: Cambridge, Cambridge University Press, $252 \mathrm{p}$.

Maniar, P.D., Piccoli, P.M., 1989, Tectonic discrimination of granitoids: Geological Society of America Bulletin, 101, 635-643.

McDowell, F.W., Mauger, R.L., 1994, K-Ar and U-Pb zircon chronology of Late Cretaceous and Tertiary magmatism in central Chihuahua State, Mexico: Geological Society of America Bulletin, 106, 118-132.

McDowell, F.W., Roldán-Quintana, J., Connelly, J.N., 2001, Duration of Late Cretaceous-early Tertiary magmatism in east-central Sonora, Mexico: Geological Society of America Bulletin, 113, 521-531.

Melgarejo i Draper, J.C., 1997, Atlas de asociaciones minerales en lámina delgada: Barcelona, Ediciones de la Universitat de Barcelona, $1074 \mathrm{p}$.

Molina-Garza, R.S., Chávez-Cabello, G., Iriondo, A., Porras-Vázquez, M.A., Terrazas-Calderón, G.D., 2008, Paleomagnetism, structure and ${ }^{40} \mathrm{Ar}{ }^{39} \mathrm{Ar}$ geochronology of the Cerro Mercado pluton, Coahuila: Implications for the timing of the Laramide orogeny in northern Mexico: Revista Mexicana de Ciencias Geológicas, 25, 284-301.

Morton-Bermea, O., 1990, Zur Petrologie des AlkaligesteinsIntrusivkomplexes der Sierra de Picachos (Nuevo Leon, Mexiko): Karlsruhe, Alemania, Universität Karlsruhe, tesis de diploma, 114 p.

Morton-Bermea, O., 1995, Petrologie, Mineralogie und Geochemie des Alkali-Intrusivkomplexes von Monclova-Candela (Mexiko): Hamburgo, Alemania, Universität Hamburg, tesis doctoral, 100 p. 
Mujica-Mondragón, M.R., Jacobo-Albarrán, J., 1983, Estudio petrogenético de las rocas ígneas y metamórficas del Altiplano Mexicano: México D.F., Instituto Mexicano del Petróleo, Proyecto C-1156, 78 p.

Nick, K., 1988, Mineralogische, geochemische und petrographische Untersuchungen in der Sierra de San Carlos (Mexiko): Karlsruhe, Alemania, Universität Karlsruhe, tesis doctoral, 167 p.

Padilla y Sánchez, R.J., 1982, Geologic evolution of the Sierra Madre Oriental between Linares, Concepción del Oro, Saltillo and Monterrey, México: Austin, Texas, University of Texas, tesis doctoral, $217 \mathrm{p}$.

Palme, H., O’Neill, H.St.C., 2003, Cosmochemical estimates of mantle composition, en Carlson, R.W. (ed.), Treatise of geochemistry, vol. 2. The mantle and core: Amsterdam, Elsevier, 1-38.

Pearce, J.A., 1983, Role of the sub-continental lithosphere in magma genesis at active continental margins, en Hawkesworth, C.J. y Norry, M.J. (eds.), Continental Basalts and Mantle Xenoliths: Cambridge, MA, Shiva Publishing Ltd., 230-249

Pearce, J.A., 1996, Sources and settings of granitic rocks: Episodes, 19, 120-125.

Pearce, J.A., Harris, N.B.W., Tindle, A.G., 1984, Trace element discrimination diagrams for the tectonic interpretation of granitic rocks: Journal of Petrology, 25, 956-983.

Pérez-Venzor, J.A., Aranda-Gómez, J.J., McDowell, F., Solorio Munguia, J.G., 1996, Geología del volcán Palo Huérfano, Guanajuato, México: Revista Mexicana de Ciencias Geológicas, 13, 174-183.

Qian, Q., Chung, S.L., Lee, T.Y., Wen, D.J., 2003, Mesozoic high-BaSr granitoids from North China: geochemical characteristics and geological implications: Terra Nova, 15, 272-278.

Ramírez-Fernández, J.A., 1996, Zur Petrogenese des Alkalikomplex der Sierra de Tamaulipas, NE Mexiko: Freiburg, Alemania, Universität Freiburg, tesis doctoral, $317 \mathrm{p}$.

Ramos-Velázquez, E., Calmus, T., Valencia, V., Iriondo, A., ValenciaMoreno, M., Bellon, H., 2008, U-Pb and ${ }^{40} \mathrm{Ar} /{ }^{39} \mathrm{Ar}$ geochronology of the coastal Sonora batholith: New insights on Laramide continental arc magmatism: Revista Mexicana de Ciencias Geológicas, 25, 314-333.

Rangin, C., Le Pichon, X., MartinezReyes, J., ArandaGarcía, M., 2008, Gravity tectonics and plate motions: The western margin of the Gulf of Mexico Introduction: Bulletin de la Société Géologique de France, 179, 107-116.

Reverdatto, V.V., Kalinin, A.S., 1980, Two models of the origin of granitoid magma and accompanying metamorphism in mobile belts of the earth's crust: Tectonophysics, 67, 101-121.

Roger, C.L., De Cserna, Z., Tavera, A.E., Ulloa, S., 1957, Geología general y depósitos de fosfatos del distrito de Concepción del Oro, estado de Zacatecas: Boletín del Instituto Nacional para la Investigación de Recursos Minerales, 38, 1-129.

Roger, C.L., Vloten, V.R., Rivera, O.J., Tavera, A.E., De Cserna, Z., 1963, Plutonic rocks of northern Zacatecas and adjacent areas, Mexico: Boletín del Consejo de Recursos Naturales no Renovables, 61, 7-10.

Rollinson, H.R., 1993, Using geochemical data: evaluation, presentation, interpretation: Essex, Inglaterra, Longman, $352 \mathrm{p}$.

Rudnick, R.L., Gao, S., 2003, Composition of the continental crust, en Rudnick, R.L. (ed.), Treatise of geochemistry Vol. 3, The crust: Amsterdam, Elsevier, 1-64.
Servicio Geológico Mexicano, 2000a, Carta geológico-minera Concepción del Oro G14-10, 1:250,000: Pachuca, Hidalgo, México, Servicio Geológico Mexicano, 1 mapa.

Servicio Geológico Mexicano, 2000b, Carta magnética de campo total Concepción del Oro G14-10, 1:250,000: Pachuca, Hidalgo, México, Servicio Geológico Mexicano, 1 mapa.

Stein, G., Lapierre, H., Monod, O., Zimmerman, J.-L., Vidal, R., 1994, Petrology of some Mexican mesozoic-cenozoic plutons: Sources and tectonic environments: Journal of South American Earth Sciences, 7, 1-7.

Tarney, J., Jones, C.E., 1994, Trace element geochemistry of orogenic igneous rocks and crustal growth models: Journal of the Geological Society, 151, 855-868.

Tristán-González, M., Torres-Hernández, J.R., 1994, Geología de la sierra de Charcas, Estado de San Luis Potosí, México: Revista Mexicana de Ciencias Geológicas, 11, 117-138.

Valencia-Moreno, M., Ruiz, J., Barton, M.D., Patchett, P.J., Zürcher, L., Hodkinson, D.G., Roldán-Quintana, J., 2001, A chemical and isotopic study of the Laramide granitic belt of northwestern Mexico: Identification of the southern edge of the North American Precambrian basement: Geological Society of America Bulletin, 113, 1409-1422.

Valencia-Moreno, M., Ruiz, J., Ochoa-Landín, L., Martínez-Serrano, R., Vargas-Navarro, P., 2003, Geochemistry of the Coastal Sonora batholith, Northwestern Mexico: Canadian Journal of Earth Sciences, 40, 819-831.

Valencia-Moreno, M., Iriondo, A., González-León, C., 2006, Temporal constraints on the eastward migration of the Late Cretaceous-early Tertiary magmatic arc of NW Mexico based on new ${ }^{40} \mathrm{Ar} /{ }^{39} \mathrm{Ar}$ hornblende geochronology of granitic rocks: Journal of South American Earth Sciences, 22, 22-38.

Verma, S.P., Torres-Alvarado, I.S., Sotelo-Rodríguez, Z.T., 2002, SINCLAS: standard igneous norm and volcanic rock classification system: Computers \& Geosciences, 28, 711-715.

Verma, S.P., Torres-Alvarado, I.S., Velasco-Tapia, F., 2003, A revised CIPW norm: Schweizerische Mineralogische und Petrographische Mitteilungen (Swiss Bulletin of Mineralogy and Petrology), 83, 197-216.

Whalen, J.B., Currie, K.L., Chappell, B.W., 1987, A-type granites: geochemical characteristics, discrimination and petrogenesis: Contributions to Mineralogy and Petrology, 95, 407-419.

Wilson, M., 1989, Igneous petrogenesis: A global tectonic approach: Londres, Chapman \& Hall, 466 p.

Manuscrito recibido: Junio 15, 2009.

Manuscrito corregido recibido: Junio 1, 2010.

Manuscrito aceptado: Julio 8, 2010. 